\title{
Momentum-space treatment of the Coulomb interaction in three-nucleon reactions with two protons
}

\author{
A. Deltuva, ${ }^{1,2, *, \dagger}$ A. C. Fonseca, ${ }^{1}$ and P. U. Sauer ${ }^{2}$ \\ ${ }^{1}$ Centro de Física Nuclear da Universidade de Lisboa, P-1649-003 Lisboa, Portugal \\ ${ }^{2}$ Institut für Theoretische Physik, Universität Hannover, D-30167 Hannover, Germany
}

(Received 31 January 2005; published 24 May 2005)

\begin{abstract}
The Coulomb interaction between two protons is included in the calculation of proton-deuteron elastic scattering, radiative proton-deuteron capture, and two-body electromagnetic disintegration of ${ }^{3} \mathrm{He}$. The hadron dynamics is based on the purely nucleonic charge-dependent (CD) Bonn potential and its realistic extension CD Bonn $+\Delta$ to a coupled-channel two-baryon potential, allowing for single virtual $\Delta$-isobar excitation. Calculations are done using integral equations in momentum space. The screening and renormalization approach is employed for including the Coulomb interaction. Convergence of the procedure is found at moderate screening radii. The reliability of the method is demonstrated. The Coulomb effect on observables is seen at low energies for the entire kinematic regime. In proton-deuteron elastic scattering at higher energies the Coulomb effect is confined to forward scattering angles; the $\Delta$-isobar effect found previously remains unchanged by the Coulomb effect. In electromagnetic reactions the Coulomb effect competes with other effects in a complicated way.
\end{abstract}

DOI: 10.1103/PhysRevC.71.054005

PACS number(s): 21.30.-x, 21.45.+v, 24.70.+s, 25.10.+s

\section{INTRODUCTION}

Experimentally, hadronic three-nucleon scattering is predominantly studied in proton-deuteron $(p d)$ reactions (i.e., in $p d$ elastic scattering and breakup). Proton and deuteron beams and targets are available, with and without polarization. The detection of charged particles yields complete experiments. In contrast, the charge-symmetric neutron-deuteron $(n d)$ reactions are much more difficult to perform, since neutron beams are scarce, neutron targets are nonexisting, and the detection of two neutrons is a complicated experimental endeavor. In electromagnetic (e.m.) reactions, proton-deuteron radiative capture has a corresponding advantage over neutron-deuteron capture and, furthermore, ${ }^{3} \mathrm{He}$ is a safer target with breakup products that are easier to detect compared with those of ${ }^{3} \mathrm{H}$.

In contrast, the Coulomb interaction between two protons is a nightmare for the theoretical description of three-nucleon reactions. The Coulomb interaction is well known, in contrast to the strong two-nucleon and three-nucleon potentials mainly studied in three-nucleon scattering. However, because of its $1 / r$ behavior, the Coulomb interaction does not satisfy the mathematical properties required for the formulation of standard scattering theory. When the theoretical description of three-particle scattering is attempted in integral form, the Coulomb interaction renders the standard equations illdefined; the kernel of the equations is noncompact. When the theoretical description is based on differential equations, the asymptotic boundary conditions for the wave function have to be numerically imposed on the trial solutions and, in the presence of the Coulomb interaction, those boundary conditions are nonstandard.

There is a long history of theoretical prescriptions for the solution of the Coulomb problem in three-particle scattering,

\footnotetext{
*Electronic address: deltuva@cii.fc.ul.pt

${ }^{\dagger}$ On leave from Institute of Theoretical Physics and Astronomy, Vilnius University, Vilnius 2600, Lithuania.
}

where different procedures are followed by the groups involved. A modified momentum-space integral equation approach is used in Refs. [1,2], whereas the configuration-space differential equation approach is used in Ref. [3] in a variational framework and in Refs. [4,5] in the framework of the Faddeev equations. There are more recent formulations [6,7] of exact scattering equations with the Coulomb interaction; however, these have not matured yet into practical applications. In addition there exist approximate schemes: The most brutal one is the description without the Coulomb interaction for the three-nucleon system with two protons at those energies and in those kinematical regimes in which the Coulomb interaction is believed to be irrelevant for observables; such an approximation has become standard in recent years [8], and, to our own guilt, we admit having used it [9]. Reference [10] extends the assumed applicability of that approximation scheme by the addition of external Coulomb correction terms to those non-Coulomb results.

In this paper our treatment of the Coulomb interaction is based on the ideas proposed in Ref. [11] for the scattering of two charged particles and extended in Ref. [12] for threeparticle scattering. The Coulomb potential is screened, the standard scattering theory for short-range potentials is used, and the obtained results are corrected for the unscreened limit. We rely on Refs. $[11,12]$ with respect to the mathematical rigor of that procedure. We constrain this paper to the description of reactions involving the $p d$ system. Thus, we leave out breakup in $p d$ scattering and three-body breakup in e.m. reactions with ${ }^{3} \mathrm{He}$. We first explain the features of our procedure to ease the understanding for the uninitiated reader and to point out differences of our treatment relative to Refs. [1,2], which also are based on Refs. [11,12].

(1) The calculations of Refs. [1,2] need improvement with respect to the hadronic interaction. Whereas the authors of Refs. [1,2] limited themselves to the use of low-rank separable potentials, we use modern two-nucleon potentials and three-nucleon forces in full without separable 


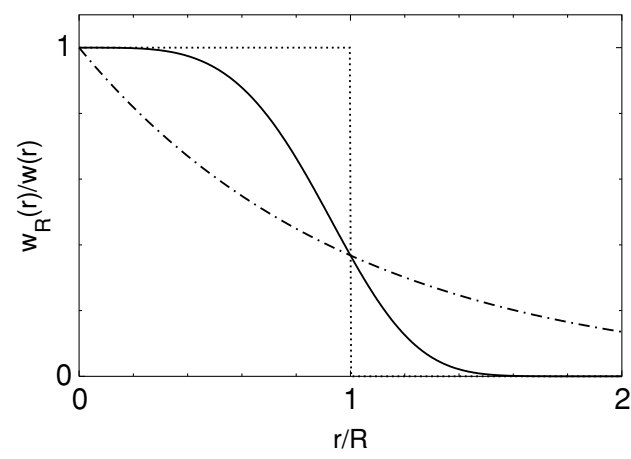

FIG. 1. Screening function $w_{R}(r) / w(r)$ as a function of the proton-proton distance $r$ for characteristic values of the parameter $n$ in Eq. (1): $n=1$ (dashed-dotted curve) corresponds to Yukawa screening, $n=4$ (solid curve) is the choice of this paper, and $n \rightarrow \infty$ (dotted curve) corresponds to a sharp cutoff.

expansion. In particular, the results of this paper are based on the purely nucleonic charge-dependent (CD) Bonn potential [13] and on its coupled-channel extension $C D$ Bonn $+\Delta[14]$, allowing for a single virtual $\Delta$-isobar excitation and fitted to the experimental data with the same degree of accuracy as the CD Bonn potential itself. In the three-nucleon system the $\Delta$ isobar mediates an effective three-nucleon force and effective two- and threenucleon currents, both consistent with the underlying effective two-nucleon force. A reliable technique [9] for solving the three-particle Alt-Grassberger-Sandhas (AGS) equation [15] without the Coulomb interaction is at our disposal. We extend that technique to include the screened Coulomb potential between the protons. Thus, the form of our three-particle equations including the screened Coulomb potential is completely different from the quasiparticle equations of two-body type solved in Refs. [1,2].

(2) We work with a Coulomb potential $w_{R}$, screened around the separation $r=R$ between two charged baryons. We choose $w_{R}$ in configuration space as

$$
w_{R}(r)=w(r) e^{-(r / R)^{n}},
$$

with the true Coulomb potential $w(r)=\alpha_{e} / r$, where $\alpha_{e} \approx 1 / 137$ is the fine structure constant and $n$ controls the smoothness of the screening. We prefer to work with a sharper screening than the Yukawa screening $(n=1)$ of Refs. $[1,2]$. We want to ensure that the screened Coulomb potential $w_{R}$ approximates well the true Coulomb one $w$ for distances $r<R$ and simultaneously vanishes rapidly for $r>R$, providing a comparatively fast convergence of the partial-wave expansion. In contrast, the sharp cutoff $(n \rightarrow \infty)$ yields an unpleasant oscillatory behavior in the momentum-space representation, leading to convergence problems. We find that values $3 \leqslant n \leqslant 6$ provide a sufficiently smooth, but at the same time a sufficiently rapid, screening around $r=R ; n=4$ is our choice for the results of this paper, unless indicated otherwise. The screening functions for different $n$ values are compared in Fig. 1, which shows that the present choice $n=4$ includes much more of the exact Coulomb potential at short distances than the Yukawa screening. For example, Yukawa screening requires a screening radius of $R=1280 \mathrm{fm}$ to approximate true Coulomb potential at $r=5 \mathrm{fm}$ as well as the present choice does with $R=$ $20 \mathrm{fm}$. That fact is the reason why Yukawa screening in Refs. [1,2] requires very large values of $R$ for convergence compared to those that the calculations of this paper will need.

(3) The screening radius $R$ is chosen to be much larger than the range of the strong interaction, which is of the order of the pion wavelength $\hbar / m_{\pi} c \approx 1.4 \mathrm{fm}$. Nevertheless, the screened Coulomb potential $w_{R}$ is of short range in the sense of scattering theory. Standard scattering theory is therefore applicable. However, the partial-wave expansion of the pair interaction requires much higher angular momenta than that of the strong two-nucleon potential alone, but not as high as one would need if Yukawa screening were used.

(4) The screening radius $R$ will always remain very small compared with the nuclear screening distances, which are of atomic scale (i.e., $10^{5} \mathrm{fm}$ ). Thus, the employed screened Coulomb potential $w_{R}$ is unable to simulate the physics of nuclear screening properly and is even poorer at modeling all features of the true Coulomb potential. Therefore $w_{R}$ is unable to yield the Coulomb scattering amplitude or the logarithmic distortion of the Coulomb wave function and, consequently, the true Coulomb phase shifts. However, the screened Coulomb potential is able to simulate the short-range modifications in the scattering amplitude. Since the Coulomb scattering amplitude and the Coulomb phase shifts are known, and their occurrence in the three-particle scattering amplitudes can be spotted, approximate calculations with screened Coulomb $w_{R}$ can be corrected for their shortcomings in a controlled way. References [11,12] give the prescription for the correction procedure that we follow here, this procedure involves the renormalization of the on-shell amplitudes to get the proper unscreened Coulomb limit.

(5) After the indicated corrections (4) are made, the predictions for observables of three-nucleon reactions have to show independence from the choice of the screening radius $R$, provided it is chosen sufficiently large. That convergence will be our internal criterion for the reliability of our Coulomb treatment.

Section II describes the practical working of the our program in detail. Section III presents some characteristic effects of the Coulomb interaction in three-nucleon reactions. Section IV gives our conclusions.

\section{TREATMENT OF COULOMB INTERACTION BETWEEN PROTONS}

Section I recalled the general idea for including the Coulomb interaction in $p d$ scattering and in related e.m. reactions by screening and renormalization. This section provides the theoretical framework on which we base our practical procedure. We are aware that the equations given here 
have been developed in Ref. [12], but their practical realization in Refs. [1,2] differs substantially from the work presented here. For completeness we rederive some of the equations and explain how we solve them; our description aims at elastic $p d$ scattering and e.m. reactions involving the $p d$ system. However, the essence of the procedure can already be well seen in proton-proton $(p p)$ scattering, which we therefore use as an illustrative example. The example is also an especially useful test, since exact results for the inclusion of the Coulomb interaction are readily available; we recover the exact results, which are easily obtainable in calculations with a sharp cutoff Coulomb potential [13].

The numerical results presented in this section refer to the coupled-channel potential CD Bonn $+\Delta$, which allows for single $\Delta$-isobar excitation. The Coulomb interaction occurs between the two protons and between the proton and the $\Delta^{+}$ in the coupled channel with an isobar.

\section{A. Proton-proton scattering}

The two protons interact through the strong potential $v$ and the Coulomb potential $w$. We introduce the full resolvent $g^{(R)}(z)$ for the auxiliary situation in which the Coulomb potential $w$ is replaced by the screened potential $w_{R}$,

$$
g^{(R)}(z)=\left(z-h_{0}-v-w_{R}\right)^{-1},
$$

where $h_{0}$ is the kinetic energy operator. The full resolvent $g^{(R)}(z)$ yields the full scattering state when acting on a plane-wave state $|\mathbf{p} v\rangle$ of relative momentum p, energy $e(p)$, and discrete two-particle quantum numbers $v$ and taking the appropriate limit $z=e(p)+i 0$. The full resolvent therefore also yields the desired $S$ matrix. The full resolvent $g^{(R)}(z)$ depends on the screening radius $R$ for the Coulomb interaction and that dependence is notationally indicated. Next, we discuss formal manipulations of the full resolvent. The resolvent can be decomposed according to

$$
g^{(R)}(z)=g_{0}(z)+g_{0}(z) t^{(R)}(z) g_{0}(z)
$$

with the free resolvent

$$
g_{0}(z)=\left(z-h_{0}\right)^{-1}
$$

and the full transition matrix

$$
t^{(R)}(z)=\left(v+w_{R}\right)+\left(v+w_{R}\right) g_{0}(z) t^{(R)}(z) .
$$

Of course, $t^{(R)}(z)$ must contain the pure Coulomb transition matrix $t_{R}(z)$ derived from the screened Coulomb potential alone:

$$
t_{R}(z)=w_{R}+w_{R} g_{0}(z) t_{R}(z) .
$$

Therefore, an alternative decomposition of the full resolvent (2b) that isolates $t_{R}(z)$ appears conceptually neater. Instead of correlating the plane-wave state $|\mathbf{p} v\rangle$ in a single step to the full scattering state by $g^{(R)}(z)$, it may be correlated first to a screened Coulomb state by the screened Coulomb potential $w_{R}$ through

$$
\begin{aligned}
& g_{R}(z)=\left(z-h_{0}-w_{R}\right)^{-1}, \\
& g_{R}(z)=g_{0}(z)+g_{0}(z) t_{R}(z) g_{0}(z) .
\end{aligned}
$$

Thus, the full resolvent can alternatively be decomposed into

$$
g^{(R)}(z)=g_{R}(z)+g_{R}(z) \tilde{t}^{(R)}(z) g_{R}(z)
$$

with the short-range operator

$$
\tilde{t}^{(R)}(z)=v+v g_{R}(z) \tilde{t}^{(R)}(z) .
$$

Equations (2b), (6), and (7) give an alternative form for the difference of transition matrices $\left[t^{(R)}(z)-t_{R}(z)\right]$, that is,

$$
t^{(R)}(z)-t_{R}(z)=\left[1+t_{R}(z) g_{0}(z)\right] \tilde{t}^{(R)}(z)\left[1+g_{0}(z) t_{R}(z)\right] .
$$

This equation is the well-known two-potential formula that achieves a clean separation of the full transition matrix $t^{(R)}(z)$ into a long-range part $t_{R}(z)$ and a short-range part $\left[t^{(R)}(z)-\right.$ $\left.t_{R}(z)\right]$. In this paper the left-hand side of Eq. (9) is calculated directly from the potentials $v$ and $w_{R}$ according to Eqs. (4) and (5). Equation (9) is only introduced by us to demonstrate that $\left[t^{(R)}(z)-t_{R}(z)\right]$, even in the infinite- $R$ limit, is a shortrange operator resulting from the short-range nature of $v$ and $\tilde{t}^{(R)}(z)$. However, on-shell, it is externally distorted owing to the screened Coulomb wave generated by $\left[1+g_{0}(z) t_{R}(z)\right]$, which together with the long-range part $t_{R}(z)$ does not have a proper limit as $R \rightarrow \infty$. This difficulty brings about the concept of renormalization of on-shell matrix elements of the operators as proposed in Refs. $[11,12]$ to recover the proper results in the unscreened Coulomb limit.

According to Refs. [11,12], the $p p$ transition amplitude $\left\langle\mathbf{p}_{f} v_{f}|t| \mathbf{p}_{i} v_{i}\right\rangle$, referring to the strong potential $v$ and the unscreened Coulomb potential $w$, is obtained via the renormalization of the on-shell $t^{(R)}(z)$ with $z=e\left(p_{i}\right)+i 0$ in the infinite- $R$ limit:

$$
\begin{aligned}
\left\langle\mathbf{p}_{f} v_{f}|t| \mathbf{p}_{i} v_{i}\right\rangle= & \lim _{R \rightarrow \infty}\left\{z_{R}^{-\frac{1}{2}}\left(p_{f}\right)\left\langle\mathbf{p}_{f} v_{f}\right| t^{(R)}\left(e\left(p_{i}\right)+i 0\right)\right. \\
& \left.\times\left|\mathbf{p}_{i} v_{i}\right\rangle z_{R}^{-\frac{1}{2}}\left(p_{i}\right)\right\}
\end{aligned}
$$

The transition amplitude $\left\langle\mathbf{p}_{f} v_{f}|t| \mathbf{p}_{i} v_{i}\right\rangle$ connects the initial and final states $\left|\mathbf{p}_{i} v_{i}\right\rangle$ and $\left|\mathbf{p}_{f} v_{f}\right\rangle, p_{f}=p_{i}$, of the considered reaction. However, Eq. (10a) as it stands is unsuitable for the numerical calculation of the full transition amplitude; instead, the split of the full transition matrix $t^{(R)}(z)$ into long- and short-range parts is more convenient. For the on-shell screened Coulomb transition matrix $t_{R}(z)$, contained in $t^{(R)}(z)$, the limit in Eq. (10a) can be carried out analytically, yielding the true Coulomb transition amplitude $\left\langle\mathbf{p}_{f} v_{f}\left|t_{C}\right| \mathbf{p}_{i} v_{i}\right\rangle$ [11], that is,

$$
\begin{aligned}
\left\langle\mathbf{p}_{f} v_{f}|t| \mathbf{p}_{i} v_{i}\right\rangle= & \left\langle\mathbf{p}_{f} v_{f}\left|t_{C}\right| \mathbf{p}_{i} v_{i}\right\rangle \\
& +\lim _{R \rightarrow \infty}\left(z _ { R } ^ { - \frac { 1 } { 2 } } ( p _ { f } ) \langle \mathbf { p } _ { f } v _ { f } | \left[t^{(R)}\left(e\left(p_{i}\right)+i 0\right)\right.\right. \\
& \left.\left.-t_{R}\left(e\left(p_{i}\right)+i 0\right)\right]\left|\mathbf{p}_{i} v_{i}\right\rangle z_{R}^{-\frac{1}{2}}\left(p_{i}\right)\right),
\end{aligned}
$$

whereas the limit for the remaining short-range part $\left[t^{(R)}(z)-\right.$ $t_{R}(z)$ ] of the transition matrix $t^{(R)}(z)$ has to be performed numerically, but it is reached with sufficient accuracy at finite screening radii $R$. In contrast to $\left\langle\mathbf{p}_{f} v_{f}\left|t_{C}\right| \mathbf{p}_{i} v_{i}\right\rangle$, the short-range part $\left[t^{(R)}(z)-t_{R}(z)\right]$ can be calculated using a partial-wave expansion of Eqs. (4) and (5). 
The renormalization factor for $R \rightarrow \infty$ is a diverging phase factor

$$
z_{R}(p)=e^{-2 i \varphi_{R}(p)}
$$

where $\varphi_{R}(p)$, though independent of the $p p$ relative orbital angular momentum $L$ in the infinite- $R$ limit, can be realized according to [11] by

$$
\varphi_{R}(p)=\sigma_{L}(p)-\eta_{L R}(p),
$$

with the diverging screened Coulomb phase shift $\eta_{L R}(p)$ corresponding to standard boundary conditions and the proper Coulomb one $\sigma_{L}(p)$ referring to the logarithmically distorted proper Coulomb boundary conditions. The form (11b) of the renormalization phase is readily understood by looking back to Eq. (9) and realizing that the external distortion generated by the screened Coulomb wave function $\left[1+g_{0}(e(p)+\right.$ $\left.i 0) t_{R}(e(p)+i 0)\right]|\mathbf{p} v\rangle$ carries, in each partial wave, the overall phase factor $e^{i \eta_{L R}(p)}$ [16]. Except for this overall phase factor, the screened Coulomb wave approximates well the unscreened one in the range required by the operator $\tilde{t}^{(R)}(z)$ in Eq. (9) for distances $r<R$. Therefore, through the renormalization, that unwanted phase factor is changed to the appropriate phase factor $e^{i \sigma_{L}(p)}$ for the unscreened Coulomb wave.

For the screened Coulomb potential of Eq. (1) the infinite- $R$ limit of $\varphi_{R}(p)$ is known analytically [11] and is given by

$$
\varphi_{R}(p)=\kappa(p)[\ln (2 p R)-C / n],
$$

where $\kappa(p)=\alpha_{e} \mu / p$ is the Coulomb parameter, $\mu$ is the reduced $p p$ mass, $C \approx 0.5772156649$ is the Euler number, and $n$ is the exponent in Eq. (1). The renormalization phase $\varphi_{R}(p)$ to be used in the actual calculations with finite screening radii $R$ is not unique, since only the infinite- $R$ limit matters, but the converged results have to show independence of the chosen form of $\varphi_{R}(p)$. According to our investigations this is indeed so. The results presented in this paper are based on the partial-wave dependent form (11b) of the renormalization factor for which we find the convergence with $R$ to be slightly faster than for (11c).

We refer to Refs. [11,12] for a rigorous justification of the renormalization procedure of Eqs. (10) and (11) and proceed here to study the numerical convergence of our predictions with increasing screening radius $R$ as a practical justification for the validity of the chosen Coulomb treatment.

The previous discussion left out the identity of the two protons. Taking the identity of the protons into account, we have to calculate the transition amplitude $\left\langle\mathbf{p}_{f} v_{f}|t| \mathbf{p}_{i} v_{i}\right\rangle$ of Eq. (10b) for antisymmetrized states. Practical results based on Eq. (10b) are shown in Figs. 2-4.

The Coulomb effect on the hadronic $p p$ phase shifts $\eta$ is most important in the ${ }^{1} S_{0}$ partial wave. The convergence with $R$ for the ${ }^{1} S_{0}$ phase shift, shown in Fig. 2, is impressive. The convergence is faster at higher energies. A screening radius of $R=20 \mathrm{fm}(10 \mathrm{fm})$ suffices for an agreement within $0.01^{\circ}$ with the exact phase shift values at all energies above $5 \mathrm{MeV}$ $(25 \mathrm{MeV})$. In contrast, to reproduce the ${ }^{1} S_{0} p p$ scattering length $a_{p p}^{C}=-7.815 \mathrm{fm}$ and the effective range $r_{p p}^{C}=$ $2.773 \mathrm{fm}$ within $0.010 \mathrm{fm}$, screening radii larger than $R=$ $100 \mathrm{fm}$ are required. In comparison to the screening function adopted in this paper, Fig. 2 also proves the convergence with

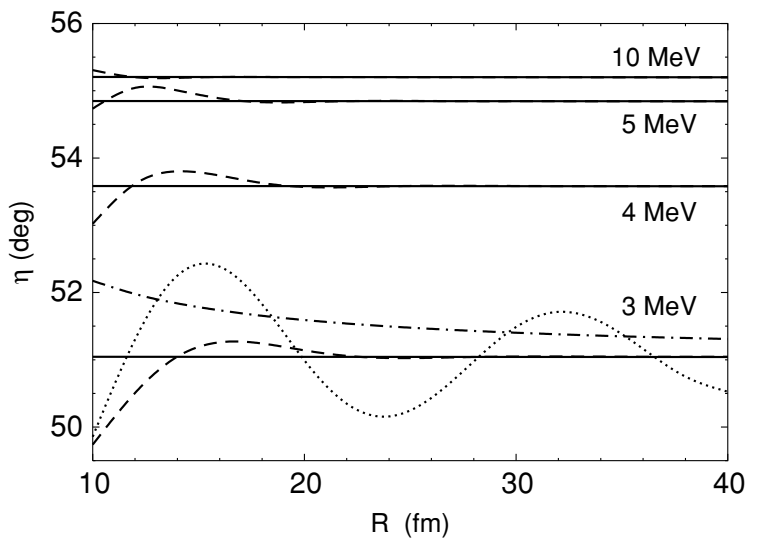

FIG. 2. Convergence of the ${ }^{1} S_{0} p p$ phase shift $\eta$ with screening radius $R$ for proton lab energies 3, 4, 5, and $10 \mathrm{MeV}$. Our results derived from Eq. (10b) and given by dashed curves are compared with exact results given by solid lines. At $3 \mathrm{MeV}$ the results obtained with Yukawa screening (dashed-dotted curve) and with a sharp cutoff (dotted curve) are also shown, demonstrating the superiority of the screening function chosen in this paper.

$R$ to be rather slow for the Yukawa screening and to be of unpleasant oscillatory behavior for a sharp cutoff.

Figure 3 exhibits the convergence of the result for the spin-averaged $p p$ differential cross section at $5-\mathrm{MeV}$ proton lab energy with increasing screening radius $R$. The screening radius $R=20 \mathrm{fm}$ appears to be sufficiently large for that energy, since, according to Fig. 3, the results for $R>20 \mathrm{fm}$ are indistinguishable from the exact Coulomb results, despite the rather fine scale of the plot. The rate of convergence seen in Fig. 3 is characteristic for all studied observables at that energy. The convergence of observables with $R$ is also faster at higher energies; beyond $25 \mathrm{MeV}$ the radius $R=10 \mathrm{fm}$ is ample enough.

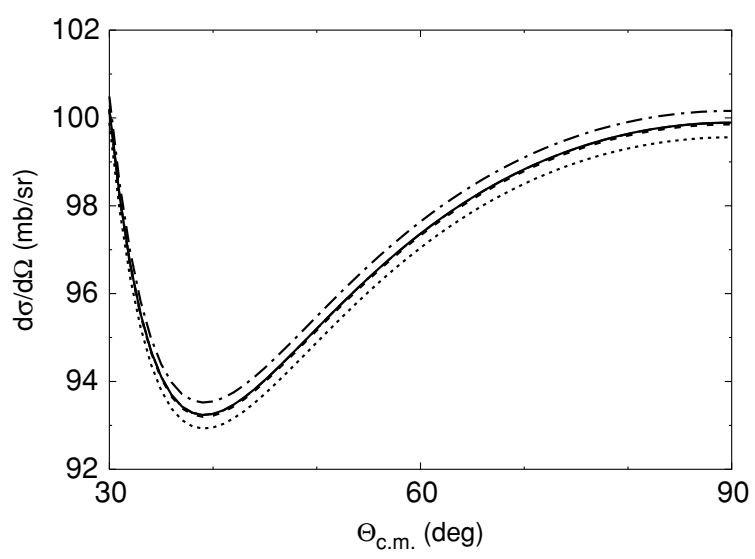

FIG. 3. Convergence of the differential cross section for $p p$ scattering at $5-\mathrm{MeV}$ proton lab energy with screening radius $R$. The cross section is shown as function of the c.m. scattering angle. Exact results given by the solid curve are compared to results with screening radii of $R=10,15$, and $20 \mathrm{fm}$, given by dotted, dashed-dotted, and dashed curves, respectively. Results obtained with $R>20 \mathrm{fm}$ are not distinguishable from the exact results. 


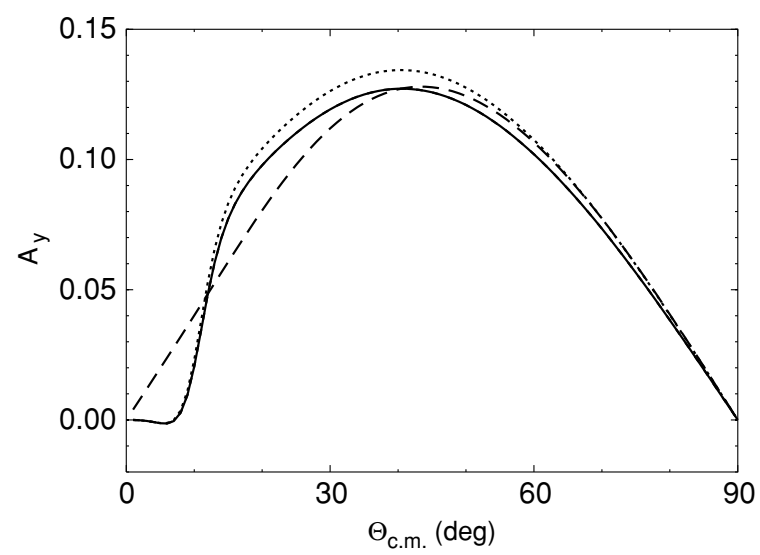

FIG. 4. Proton analyzing power for $p p$ scattering at $100-\mathrm{MeV}$ proton lab energy as a function of the c.m. scattering angle. Exact results, given by the solid curve, are indistinguishable from the results of our Coulomb treatment with screening radius $R \geqslant 10 \mathrm{fm}$; the dashed curve corresponds to no Coulomb results, and the dotted curve to the Coulomb externally corrected approximation.

Figure 4 shows the proton analyzing power results for $p p$ scattering at $100-\mathrm{MeV}$ proton lab energy. The results are converged with respect to screening and the exact results are compared with two approximations, labeled no-Coulomb and Coulomb externally corrected: In the no-Coulomb approximation, the Coulomb interaction is omitted completely; in the Coulomb externally corrected approximation, the Coulomb scattering amplitude is added to the no-Coulomb one, the latter being modified for the external Coulomb distortion by multiplication with the Coulomb phase factors $e^{i \sigma_{L}(p)}$ in the initial and final states [10]. In fact, the Coulomb externally corrected approximation coincides with a calculation with a screened Coulomb interaction, whose screening radius is taken to be zero for the short-range part of the transition amplitude. Whereas the results converged with respect to screening and the exact results are indistinguishable in Fig. 4, the noCoulomb and Coulomb externally corrected approximations are pretty poor even at $100 \mathrm{MeV}$. Note that for the observable of Fig. 3 the results for both approximations lie beyond the scale of that plot.

The results presented in Figs. 3 and 4 are characteristic for all observables studied. We conclude that the method employed for the inclusion of the Coulomb interaction in $p p$ scattering works satisfactorily. We see convergence with increasing screening radius $R$ at moderate values. The convergence in $R$ is more rapid for higher scattering energies; $R=10 \mathrm{fm}$ is sufficient for proton lab energies above $25 \mathrm{MeV}$, whereas the screening radius is to be increased beyond $20 \mathrm{fm}$ for energies below $5 \mathrm{MeV}$. We also note that the convergence in $R$ is considerably slower for Yukawa screening and is of oscillatory behavior for a sharp cutoff. The exact Coulomb results are correctly approached by the method employed with satisfactory accuracy, unlike the no-Coulomb or the Coulomb externally corrected approximations. The method we use, based on the ideas of Refs. [11,12], encourages us to carry it over to elastic $p d$ scattering as Refs. [1,2] did and to e.m. reactions involving the $p d$ system.

\section{B. Elastic proton-deuteron scattering}

This section carries over the treatment of the Coulomb interaction, given in Sec. II A for $p p$ scattering, to elastic $p d$ scattering. It establishes a theoretical procedure leading to a calculational scheme.

Each pair of nucleons $(\beta \gamma)$ interacts through the strong coupled-channel potential $v_{\alpha}$ and the Coulomb potential $w_{\alpha}$. We assume that $w_{\alpha}$ acts formally between all pairs $(\beta \gamma)$ of particles, but it is nonzero only for states with two-charged baryons (i.e., $p p$ and $p \Delta^{+}$states). We introduce the full resolvent $G^{(R)}(Z)$ for the auxiliary situation in which the Coulomb potential $w_{\alpha}$ is screened with a screening radius $R$, with $w_{\alpha}$ being replaced by $w_{\alpha R}$ :

$$
G^{(R)}(Z)=\left(Z-H_{0}-\sum_{\sigma} v_{\sigma}-\sum_{\sigma} w_{\sigma R}\right)^{-1},
$$

where $H_{0}$ is the three-particle kinetic energy operator. The full resolvent yields the full $p d$ scattering state when acting on the channel state $\left|\phi_{\alpha}(\mathbf{q}) v_{\alpha}\right\rangle$ of relative $p d$ momentum $\mathbf{q}$, energy $E_{\alpha}(q)$, and additional discrete quantum numbers $v_{\alpha}$ and taking the appropriate limit $Z=E_{\alpha}(q)+i 0$. The full resolvent therefore also yields the desired $S$ matrix. The full resolvent $G^{(R)}(Z)$ depends on the screening radius $R$ for the Coulomb interaction and that dependence is notationally indicated; the same will be done for operators related to $G^{(R)}(Z)$. The full resolvent $G^{(R)}(Z)$, following standard AGS notation [15] of three-particle scattering, may be decomposed into channel resolvents:

$$
G_{\alpha}^{(R)}(Z)=\left(Z-H_{0}-v_{\alpha}-w_{\alpha R}\right)^{-1},
$$

where, in $p d$ channels $\alpha, w_{\alpha R}=0$, and into the full multichannel three-particle transition matrix $U_{\beta \alpha}^{(R)}(Z)$ according to

$$
G^{(R)}(Z)=\delta_{\beta \alpha} G_{\alpha}^{(R)}(Z)+G_{\beta}^{(R)}(Z) U_{\beta \alpha}^{(R)}(Z) G_{\alpha}^{(R)}(Z) .
$$

The full multichannel transition matrix satisfies the AGS equation [15]

$$
U_{\beta \alpha}^{(R)}(Z)=\bar{\delta}_{\beta \alpha} G_{0}^{-1}(Z)+\sum_{\sigma} \bar{\delta}_{\beta \sigma} T_{\sigma}^{(R)}(Z) G_{0}(Z) U_{\sigma \alpha}^{(R)}(Z),
$$

where the two-particle transition matrix is derived from the full channel interaction $v_{\alpha}+w_{\alpha R}$, that is,

$T_{\alpha}^{(R)}(Z)=\left(v_{\alpha}+w_{\alpha R}\right)+\left(v_{\alpha}+w_{\alpha R}\right) G_{0}(Z) T_{\alpha}^{(R)}(Z)$,

where $G_{0}(Z)=\left(Z-H_{0}\right)^{-1}$ is the free resolvent and $\bar{\delta}_{\beta \alpha}=$ $1-\delta_{\beta \alpha}$. Of course, the full multichannel transition matrix $U_{\beta \alpha}^{(R)}(Z)$ must contain the pure Coulomb transition matrix $T_{\alpha R}^{\text {c.m. }}(Z)$ derived from the screened Coulomb potential $W_{\alpha R}^{\text {c.m. }}$ between the spectator proton and the center of mass (c.m.) of the remaining neutron-proton $(n p)$ pair in channel $\alpha$, that is,

$$
T_{\alpha R}^{\mathrm{c} . \mathrm{m}}(Z)=W_{\alpha R}^{\mathrm{c} . \mathrm{m}}+W_{\alpha R}^{\mathrm{c} . \mathrm{m}} G_{\alpha}^{(R)}(Z) T_{\alpha R}^{\mathrm{c} . \mathrm{m}}(Z),
$$

with the pd channel being one of those channels $\alpha$. The same screening function is used for both Coulomb potentials $w_{\alpha R}$ and $W_{\alpha R}^{\text {c.m. }}$.

As we have done in Sec. II A, an alternative decomposition of the full resolvent, which appears conceptually neater for the 
purpose of elastic $p d$ scattering, may be developed based on the following idea. Instead of correlating the plane-wave channel state $\left|\phi_{\alpha}(\mathbf{q}) v_{\alpha}\right\rangle$ in a single step to the full scattering state by $G^{(R)}(Z)$, it may be correlated first to a screened Coulomb state of proton and deuteron by the screened Coulomb potential

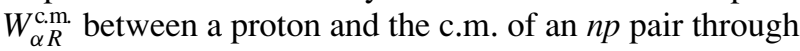

$$
\begin{aligned}
& G_{\alpha R}(Z)=\left(Z-H_{0}-v_{\alpha}-w_{\alpha R}-W_{\alpha R}^{\mathrm{c} . \mathrm{m}}\right)^{-1}, \\
& G_{\alpha R}(Z)=G_{\alpha}^{(R)}(Z)+G_{\alpha}^{(R)}(Z) T_{\alpha R}^{\mathrm{c} . \mathrm{m}}(Z) G_{\alpha}^{(R)}(Z),
\end{aligned}
$$

where, in each channel $\alpha, w_{\alpha R}$ and $W_{\alpha R}^{\text {c.m. }}$ are never simultaneously present: When $\alpha$ corresponds to a $p p$ pair, $w_{\alpha R}$ is present and $W_{\alpha R}^{\text {c.m. }}=0$; when $\alpha$ denotes an $n p$ pair, $w_{\alpha R}=0$ and $W_{\alpha R}^{\text {c.m. }}$ is present. Thus, the full resolvent can alternatively be decomposed into

$$
\begin{aligned}
G^{(R)}(Z)= & \delta_{\beta \alpha} G_{\alpha R}(Z)+G_{\beta R}(Z) \tilde{U}_{\beta \alpha}^{(R)}(Z) G_{\alpha R}(Z), \\
G^{(R)}(Z)= & \delta_{\beta \alpha} G_{\alpha}^{(R)}(Z)+G_{\beta}^{(R)}(Z) \delta_{\beta \alpha} T_{\alpha R}^{\text {c.m. }}(Z) G_{\alpha}^{(R)}(Z) \\
& +G_{\beta}^{(R)}(Z)\left\{\left[1+T_{\beta R}^{\text {c.m. }}(Z) G_{\beta}^{(R)}(Z)\right] \tilde{U}_{\beta \alpha}^{(R)}(Z)\right. \\
& \left.\times\left[1+G_{\alpha}^{(R)}(Z) T_{\alpha R}^{\text {c.m. }}(Z)\right]\right\} G_{\alpha}^{(R)}(Z),
\end{aligned}
$$

where the operator $\tilde{U}_{\beta \alpha}^{(R)}(Z)$ may be calculated through the integral equation

$$
\begin{aligned}
\tilde{U}_{\beta \alpha}^{(R)}(Z)= & \bar{\delta}_{\beta \alpha}\left[G_{\alpha R}^{-1}(Z)+v_{\alpha}\right]+\delta_{\beta \alpha} \mathcal{W}_{\alpha R} \\
& +\sum_{\sigma}\left(\bar{\delta}_{\beta \sigma} v_{\sigma}+\delta_{\beta \sigma} \mathcal{W}_{\beta R}\right) G_{\sigma R}(Z) \tilde{U}_{\sigma \alpha}^{(R)}(Z),
\end{aligned}
$$

which is driven by the strong potential $v_{\alpha}$ and the potential of three-nucleon nature $\mathcal{W}_{\alpha R}=\sum_{\sigma}\left(\bar{\delta}_{\alpha \sigma} w_{\sigma R}-\delta_{\alpha \sigma} W_{\sigma R}^{\text {c.m. }}\right)$. This potential $\mathcal{W}_{\alpha R}$ accounts for the difference between the direct pp Coulomb interaction and the one that takes place between the proton and the c.m. of the remaining bound as well as unbound $n p$ pair. When calculated between on-shell screened $p d$ Coulomb states, $\tilde{U}_{\beta \alpha}^{(R)}(Z)$ is of short range, even in the infinite- $R$ limit. Equation (18b), together with Eq. (14), gives an alternative form for the difference of the transition matrices $\left[U_{\beta \alpha}^{(R)}(Z)-\delta_{\beta \alpha} T_{\alpha R}^{\mathrm{c} . \mathrm{m} .}(Z)\right]$, that is

$$
\begin{aligned}
U_{\beta \alpha}^{(R)}(Z)-\delta_{\beta \alpha} T_{\alpha R}^{\mathrm{cm} .}(Z)= & {\left[1+T_{\beta R}^{\mathrm{c} . \mathrm{m}}(Z) G_{\beta}^{(R)}(Z)\right] \tilde{U}_{\beta \alpha}^{(R)}(Z) } \\
& \times\left[1+G_{\alpha}^{(R)}(Z) T_{\alpha R}^{\mathrm{c} . \mathrm{m} .}(Z)\right] .
\end{aligned}
$$

Though we calculate that difference directly from the poten-

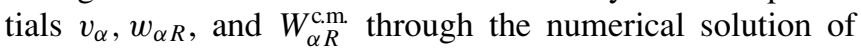
Eqs. (15) and (16), Eq. (20) demonstrates that for initial and final $p d$ states $\left[U_{\beta \alpha}^{(R)}(Z)-\delta_{\beta \alpha} T_{\alpha R}^{\mathrm{c} . \mathrm{m} .}(Z)\right]$ is a short-range operator owing to the nature of $\tilde{U}_{\beta \alpha}^{(R)}(Z)$ as previously discussed, but it is externally distorted owing to the screened Coulomb wave generated by $\left[1+G_{\alpha}^{(R)}(Z) T_{\alpha R}^{\text {c.m. }}(Z)\right]$. Thus, Eq. (20) achieves a clean separation of the full on-shell transition matrix $U_{\beta \alpha}^{(R)}(Z)$ into the long-range part $\delta_{\beta \alpha} T_{\alpha R}^{\text {c.m. }}(Z)$ and the short-range part $\left[U_{\beta \alpha}^{(R)}(Z)-\delta_{\beta \alpha} T_{\alpha R}^{\text {c.m. }}(Z)\right]$. On-shell, both parts do not have a proper limit as $R \rightarrow \infty$. To obtain the results appropriate for the unscreened Coulomb limit, they need to be renormalized as was done for the corresponding amplitudes for $p p$ scattering in Sec. II A.

According to Refs. [11,12], the full $p d$ transition amplitude for initial and final states $\left|\phi_{\alpha}\left(\mathbf{q}_{i}\right) v_{\alpha_{i}}\right\rangle$ and $\left|\phi_{\beta}\left(\mathbf{q}_{f}\right) v_{\beta_{f}}\right\rangle, q_{f}=$ $q_{i}$, referring to the strong potential $v_{\alpha}$ and the unscreened Coulomb potential $w_{\alpha}$, is obtained via the renormalization of the on-shell multichannel transition matrix $U_{\beta \alpha}^{(R)}(Z)$ with $Z=E_{\alpha}\left(q_{i}\right)+i 0$ in the infinite- $R$ limit:

$$
\begin{aligned}
& \left\langle\phi_{\beta}\left(\mathbf{q}_{f}\right) v_{\beta_{f}}\left|U_{\beta \alpha}\right| \phi_{\alpha}\left(\mathbf{q}_{i}\right) v_{\alpha_{i}}\right\rangle=\lim _{R \rightarrow \infty}\left[\mathcal{Z}_{R}^{-\frac{1}{2}}\left(q_{f}\right)\left\langle\phi_{\beta}\left(\mathbf{q}_{f}\right) v_{\beta_{f}}\right|\right. \\
& \left.\quad \times U_{\beta \alpha}^{(R)}\left(E_{\alpha}\left(q_{i}\right)+i 0\right)\left|\phi_{\alpha}\left(\mathbf{q}_{i}\right) v_{\alpha_{i}}\right| \mathcal{Z}_{R}^{-\frac{1}{2}}\left(q_{i}\right)\right] .
\end{aligned}
$$

As for $p p$ scattering, splitting the full on-shell multichannel transition matrix $U_{\beta \alpha}^{(R)}(Z)$ into long- and short-range parts is most convenient. For the screened Coulomb transition matrix $T_{\alpha R}^{\mathrm{c} . \mathrm{m} .}(Z)$, contained in $U_{\beta \alpha}^{(R)}(Z)$, the limit in Eq. (21a) can be carried out analytically, yielding the proper Coulomb transition amplitude $\left\langle\phi_{\beta}\left(\mathbf{q}_{f}\right) v_{\beta_{f}}\left|T_{\alpha C}^{\mathrm{c} . \mathrm{m}}\right| \phi_{\alpha}\left(\mathbf{q}_{i}\right) v_{\alpha_{i}}\right\rangle[11,12]$, that is,

$$
\begin{gathered}
\left\langle\phi_{\beta}\left(\mathbf{q}_{f}\right) v_{\beta_{f}}\left|U_{\beta \alpha}\right| \phi_{\alpha}\left(\mathbf{q}_{i}\right) v_{\alpha_{i}}\right\rangle=\delta_{\beta \alpha}\left\langle\phi_{\beta}\left(\mathbf{q}_{f}\right) v_{\beta_{f}}\left|T_{\alpha C}^{\mathrm{c} . \mathrm{m}}\right| \phi_{\alpha}\left(\mathbf{q}_{i}\right) v_{\alpha_{i}}\right\rangle \\
\quad+\lim _{R \rightarrow \infty}\left(\mathcal { Z } _ { R } ^ { - \frac { 1 } { 2 } } ( q _ { f } ) | \phi _ { \beta } ( \mathbf { q } _ { f } ) v _ { \beta _ { f } } | \left[U_{\beta \alpha}^{(R)}\left(E_{\alpha}\left(q_{i}\right)+i 0\right)\right.\right. \\
\left.\left.\quad-\delta_{\beta \alpha} T_{\alpha R}^{\mathrm{c} . \mathrm{m}}\left(E_{\alpha}\left(q_{i}\right)+i 0\right)\right]\left|\phi_{\alpha}\left(\mathbf{q}_{i}\right) v_{\alpha_{i}}\right| \mathcal{Z}_{R}^{-\frac{1}{2}}\left(q_{i}\right)\right) . \quad(21 \mathrm{~b})
\end{gathered}
$$

The limit for the remaining part $\left[U_{\beta \alpha}^{(R)}(Z)-\delta_{\beta \alpha} T_{\alpha R}^{\text {c.m. }}(Z)\right]$ of the multichannel transition matrix has to be performed numerically, but, owing to the short-range nature of that part, it is reached with sufficient accuracy at finite screening radii $R$, and furthermore, $\left[U_{\beta \alpha}^{(R)}(Z)-\delta_{\beta \alpha} T_{\alpha R}^{\mathrm{c} . \mathrm{m}}(Z)\right]$ can be calculated using a partial-wave expansion.

In close analogy with $p p$ scattering, the renormalization factor for $R \rightarrow \infty$ is a diverging phase factor

$$
\mathcal{Z}_{R}(q)=e^{-2 i \phi_{R}(q)},
$$

where $\phi_{R}(q)$, though independent of the $p d$ relative angular momentum $l$ in the infinite- $R$ limit, is realized by

$$
\phi_{R}(q)=\sigma_{l}(q)-\eta_{l R}(q),
$$

with the diverging screened Coulomb $p d$ phase shift $\eta_{l R}(q)$ corresponding to standard boundary conditions and the proper Coulomb one $\sigma_{l}(q)$ referring to the logarithmically distorted proper Coulomb boundary conditions. In analogy to $p p$ scattering the form (22b) of the renormalization phase is readily understood by looking back to Eq. (20). For the screened Coulomb potential of Eq. (1) the infinite- $R$ limit of $\phi_{R}(q)$ is known analytically and is given by

$$
\phi_{R}(q)=\kappa(q)[\ln (2 q R)-C / n],
$$

where $\kappa(q)=\alpha_{e} M / q$ is the $p d$ Coulomb parameter and $M$ is the reduced $p d$ mass. The form of the renormalization phase $\phi_{R}(q)$ to be used in the actual calculations with finite screening radii $R$ is not unique, but, as in Sec. II A, the converged results show independence of the chosen form of $\phi_{R}(q)$. The results presented in this paper are based on the partial-wave dependent form (22b) of the renormalization factor for which we find the convergence with $R$ to be slightly faster than for (22c).

Again we refer to Refs. [11,12] for a rigorous justification of the correction procedure of Eqs. (21) and (22) and proceed here to study the numerical convergence of our predictions with increasing $R$.

We choose an isospin description for the three baryons involved in three-nucleon scattering. In the isospin formalism 
the isospin $T$ of the interacting pair and the isospin $t$ of the spectator are coupled to the total isospin $\mathcal{T}$ with the projection $\mathcal{M}_{\mathcal{T}}$. Because of the hadronic charge dependence together with the screened Coulomb interaction in $p p$ and $p \Delta^{+}$pair states (i.e., in states with isospin $\left|T M_{T}\right\rangle=|11\rangle$ ), the two-baryon transition matrix $T_{\alpha}^{(R)}(Z)$ becomes an operator coupling total isospin $\mathcal{T}=\frac{1}{2}$ and $\mathcal{T}=\frac{3}{2}$ states according to

$$
\begin{aligned}
& \left\langle\left(T^{\prime} t^{\prime}\right) \mathcal{T}^{\prime} \mathcal{M}_{\mathcal{T}}^{\prime}\left|T_{\alpha}^{(R)}(Z)\right|(T t) \mathcal{T} \mathcal{M}_{\mathcal{T}}\right\rangle \\
& =\delta_{T^{\prime} T} \delta_{t^{\prime} t} \delta_{\mathcal{M}_{\mathcal{T}}^{\prime} \mathcal{M}_{\mathcal{T}}} \sum_{M_{T} m_{t}}\left\langle T M_{T} t m_{t} \mid \mathcal{T}^{\prime} \mathcal{M}_{\mathcal{T}}\right\rangle \\
& \quad \times\left\langle T M_{T}\left|T_{\alpha}^{(R)}(Z)\right| T M_{T}\right\rangle\left\langle T M_{T} t m_{t} \mid \mathcal{T} \mathcal{M}_{\mathcal{T}}\right\rangle .
\end{aligned}
$$

Because of the isospin formulation, the nucleons are therefore considered identical. However, the discussion has left out the identity of nucleons till now. Instead of the transition amplitude of Eq. (21b) we therefore have to use the properly symmetrized form

$$
\begin{gathered}
\left\langle\phi_{\alpha}\left(\mathbf{q}_{f}\right) v_{\alpha_{f}}|U| \phi_{\alpha}\left(\mathbf{q}_{i}\right) v_{\alpha_{i}}\right\rangle=\sum_{\sigma}\left\langle\phi_{\alpha}\left(\mathbf{q}_{f}\right) v_{\alpha_{f}}\left|U_{\alpha \sigma}\right| \phi_{\sigma}\left(\mathbf{q}_{i}\right) v_{\sigma_{i}}\right\rangle, \\
\left\langle\phi_{\alpha}\left(\mathbf{q}_{f}\right) v_{\alpha_{f}}|U| \phi_{\alpha}\left(\mathbf{q}_{i}\right) v_{\alpha_{i}}\right\rangle=\left\langle\phi_{\alpha}\left(\mathbf{q}_{f}\right) v_{\alpha_{f}}\left|T_{\alpha C}^{\mathrm{c} . \mathrm{m}}\right| \phi_{\alpha}\left(\mathbf{q}_{i}\right) v_{\alpha_{i}}\right\rangle \\
\quad+\lim _{R \rightarrow \infty}\left(\mathcal { Z } _ { R } ^ { - \frac { 1 } { 2 } } ( q _ { f } ) \langle \phi _ { \alpha } ( \mathbf { q } _ { f } ) v _ { \alpha _ { f } } | \left[U^{(R)}\left(E_{\alpha}\left(q_{i}\right)+i 0\right)\right.\right. \\
\left.\left.-T_{\alpha R}^{\mathrm{c} . \mathrm{m} .}\left(E_{\alpha}\left(q_{i}\right)+i 0\right)\right]\left|\phi_{\alpha}\left(\mathbf{q}_{i}\right) v_{\alpha_{i}}\right| \mathcal{Z}_{R}^{-\frac{1}{2}}\left(q_{i}\right)\right)
\end{gathered}
$$

with $U^{(R)}(Z)=U_{\alpha \alpha}^{(R)}(Z)+U_{\alpha \beta}^{(R)}(Z) P_{231}+U_{\alpha \gamma}^{(R)}(Z) P_{312}$ for the calculation of observables, with $(\alpha \beta \gamma)$ being cyclic and $P_{231}$ and $P_{312}$ being the two cyclic permutations of $(\alpha \beta \gamma)$. $U^{(R)}(Z)$ satisfies the standard symmetrized form of the integral equation (15a), that is,

$$
U^{(R)}(Z)=P G_{0}^{-1}(Z)+P T_{\alpha}^{(R)}(Z) G_{0}(Z) U^{(R)}(Z)
$$

with $P=P_{231}+P_{312}$.

The practical implementation of the outlined calculational scheme faces a technical difficulty. We solve Eq. (25) in a partial-wave basis. The partial-wave expansion of the screened Coulomb potential converges rather slowly. The problem does not occur in $p p$ scattering, since there the partial waves with different two-baryon total angular momentum $I$ are not coupled and the maximal $I$ required for $\left[t^{(R)}(z)-t_{R}(z)\right]$ is determined according to Eqs. (8) and (9) by the range of the hadronic potential $v$. However, in the calculation of $U^{(R)}(Z)$ all two-baryon partial waves are coupled dynamically; the required maximal $I$ is determined by the range of the screened Coulomb potential and is considerably higher than that required for the hadronic potential alone. In this context, the perturbation theory for higher two-baryon partial waves developed in Ref. [17] is a very efficient and reliable technical tool for treating the screened Coulomb interaction in high partial waves. Furthermore, in practical calculations we split the difference of the transition matrices in Eq. (24b) into two parts with different partial-wave convergence properties,

$$
\begin{aligned}
U^{(R)}(Z)-T_{\alpha R}^{\mathrm{c} . \mathrm{m}}(Z)= & {\left[U^{(R)}(Z)-P T_{\alpha R}(Z) P\right] } \\
& -\left[T_{\alpha R}^{\mathrm{c} . \mathrm{m}}(Z)-P T_{\alpha R}(Z) P\right],
\end{aligned}
$$

where $T_{\alpha R}(Z)$ is the two-baryon screened Coulomb transition matrix derived from $w_{\alpha R}$ alone and hidden in $T_{\alpha}^{(R)}(Z)$ according to Eq. (15b). The term $P T_{\alpha R}(Z) P$ is the remainder of the three-body operator $U^{(R)}(Z)-P G_{0}^{-1}(Z)$ in the absence of the strong force, and it is contained in $U^{(R)}(Z)$ as the most important Coulomb contribution; the difference $\left[U^{(R)}(Z)-P T_{\alpha R}(Z) P\right]$ converges with respect to included two-baryon states considerably faster than $U^{(R)}(Z)$ alone. The term $\left[T_{\alpha R}^{\text {c.m. }}(Z)-P T_{\alpha R}(Z) P\right]$ accounts for the off-c.m. $p d$ screened Coulomb interaction and converges rather slowly, but including very high partial waves is much easier to do than for $\left[U^{(R)}(Z)-P T_{\alpha R}(Z) P\right]$. We vary the dividing line between partial waves included exactly and perturbatively in $U^{(R)}(Z)$ as well as angular momentum cutoffs for both terms in Eq. (26) to test the convergence and thereby establish the validity of the procedure. The problem of high partial waves does not occur in Refs. [1,2], since the authors use the quasiparticle formalism and work with equations of two-body type in which the partial-wave decomposition has to be performed only with respect to the relative motion of the spectator particle and the correlated pair. Technical limitations restricted the authors of Refs. [1,2] to using low-rank separable potentials for the hadronic interaction and approximating the two-proton screened Coulomb transition matrix by the potential. In contrast, we work with a realistic hadronic interaction (CD Bonn or CD Bonn $+\Delta$ ) without separable approximation, and we never approximate the energy-dependent pair transition matrix for screened Coulomb $T_{\alpha R}(Z)$ by the potential $w_{\alpha R}$.

With respect to the partial-wave expansion in the actual calculations of this paper, we obtain fully converged results by taking into account the screened Coulomb interaction in two-baryon partial waves with pair orbital angular momentum $L \leqslant 13$ for the first term in Eq. (26) and with $L \leqslant 25$ for the second term; orbital angular momenta $L \geqslant 7$ can safely be treated perturbatively. These values refer to the screening radius $R=25 \mathrm{fm}$; for smaller screening radii the convergence in orbital angular momentum is faster. The hadronic interaction is taken into account in two-baryon partial waves with total angular momentum $I \leqslant 5$. Both three-baryon total isospin $\mathcal{T}=$ $\frac{1}{2}$ and $\mathcal{T}=\frac{3}{2}$ states are included. The maximal three-baryon total angular momentum $\mathcal{J}$ considered is $\frac{31}{2}$.

Figures 5 and 6 show the convergence of our method with increasing screening radius $R$ according to Eq. (24b). The comparison with the no-Coulomb results (dashed curves), used till now by us when accounting for $p d$ data, gives the size of the Coulomb effect. First we concentrate on 3-MeV proton lab energy, the lowest energy considered in this paper. As examples we show the differential cross section, the nucleon analyzing power $A_{y}(N)$, which has the most critical convergence behavior according to Refs. [2,18], and the deuteron tensor analyzing power $T_{21}$, the most slowly converging observable at $3-\mathrm{MeV}$ proton lab energy according to our experience. Nevertheless, the convergence is impressive even for those worst cases: Only $T_{21}$, shown in Fig. 6, requires a screening radius $R>15 \mathrm{fm}$. Convergence is more rapid at higher energies, as demonstrated in Fig. 6 for the deuteron tensor analyzing power $T_{21}$ at 3 -and $10-\mathrm{MeV}$ proton lab energy. The observed convergence strongly suggests the reliability of the chosen Coulomb treatment. Furthermore, a forthcoming paper [19] makes a detailed comparison between the results obtained by the present technique and those of 


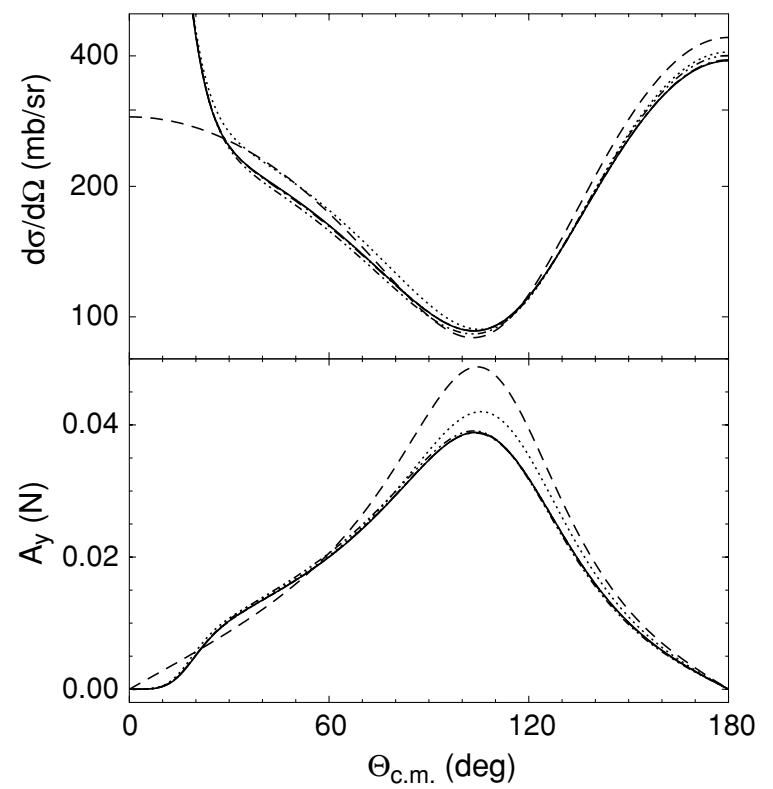

FIG. 5. Convergence of the differential cross section and of the proton analyzing power $A_{y}(N)$ for $p d$ elastic scattering at $3-\mathrm{MeV}$ proton lab energy with screening radius $R$. The observables are shown as functions of the c.m. scattering angle. The hadronic potential is CD Bonn $+\Delta$. Results obtained with screening radii of $R=5 \mathrm{fm}$ (dotted curves), $10 \mathrm{fm}$ (dashed-double-dotted curves), $15 \mathrm{fm}$ (dasheddotted curves), $20 \mathrm{fm}$ (double-dashed-dotted curves), and $25 \mathrm{fm}$ (solid curves) are compared. Results without the Coulomb interaction (dashed curves) are given as reference for the size of the Coulomb effect.

Ref. [3] obtained from the variational solution of the threenucleon Schrödinger equation in configuration space with the inclusion of an unscreened Coulomb potential between the protons and imposing the proper Coulomb boundary conditions explicitly. The agreement, across the board, between the results derived from two entirely different methods, clearly indicates that both techniques for including the Coulomb interaction are reliable; this is another justification for the technique used in this paper.

As in Fig. 4 for $p p$ scattering, Fig. 7 compares predictions including the Coulomb interaction with results from traditional approximate treatments, which were labeled before as no-Coulomb and Coulomb externally corrected. As already known from Refs. [2,18] both approximations are unsatisfactory at low energies. At higher energies the Coulomb effect is confined more and more to the forward direction; the no-Coulomb treatment fails there, whereas the Coulomb externally corrected approximation is usually not accurate enough for larger scattering angles.

The Coulomb effects seen and their physics implications are discussed in Sec. III.

\section{Radiative capture and two-body e.m. disintegration of the three-nucleon bound state}

For the description of the considered e.m. processes the matrix element $\left\langle\psi_{\alpha}^{(-)}\left(\mathbf{q}_{f}\right) v_{\alpha_{f}}\left|j^{\mu}\left(\mathbf{Q}, \mathbf{K}_{+}\right)\right| B\right\rangle$ of the e.m.

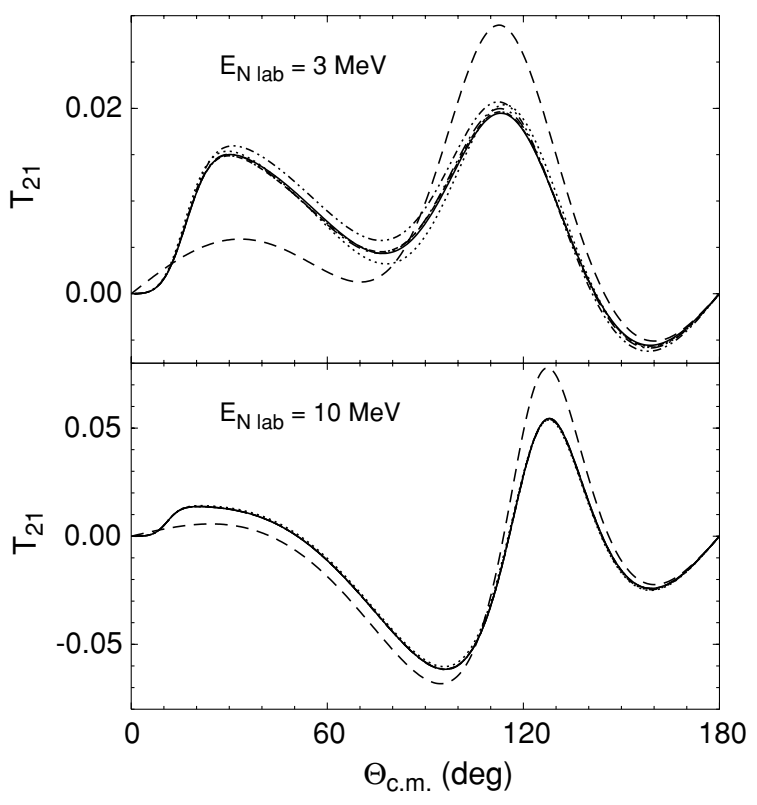

FIG. 6. Convergence of the deuteron tensor analyzing power $T_{21}$ for $p d$ elastic scattering at 3- and $10-\mathrm{MeV}$ proton lab energy with screening radius $R$. The observable is shown as function of the c.m. scattering angle. The curves are explained in the caption of Fig. 5.

current operator between the three-nucleon bound state and the $p d$ scattering state has to be calculated. The calculation of that matrix element without the Coulomb interaction is discussed in great length in Refs. [20,21]. This subsection only discusses the modification arising from the inclusion of the Coulomb interaction between the charged baryons. The Coulomb interaction is included as a screened potential and the dependence of the bound and scattering states [i.e., $\left|B^{(R)}\right\rangle$ and $\left.\left|\psi_{\alpha}^{( \pm)(R)}\left(\mathbf{q}_{f}\right) v_{\alpha_{f}}\right\rangle\right]$ on the screening radius $R$ is notationally made explicit. In analogy to $p d$ scattering, the current matrix element referring to the unscreened Coulomb potential is obtained via renormalization of the matrix element referring to the screened Coulomb potential in the infinite- $R$ limit:

$$
\begin{aligned}
& \left\langle\psi_{\alpha}^{(-)}\left(\mathbf{q}_{f}\right) v_{\alpha_{f}}\left|j^{\mu}\left(\mathbf{Q}, \mathbf{K}_{+}\right)\right| B\right\rangle \\
& \quad=\lim _{R \rightarrow \infty} \mathcal{Z}_{R}^{-\frac{1}{2}}\left(q_{f}\right)\left\langle\psi_{\alpha}^{(-)(R)}\left(\mathbf{q}_{f}\right) v_{\alpha_{f}}\left|j^{\mu}\left(\mathbf{Q}, \mathbf{K}_{+}\right)\right| B^{(R)}\right\rangle .
\end{aligned}
$$

As for $p d$ scattering, the practical results presented in this paper are based on the partial-wave dependent form of the renormalization factor (22b). Owing to the short-range nature of $j^{\mu}\left(\mathbf{Q}, \mathbf{K}_{+}\right)\left|B^{(R)}\right\rangle$ the limit $R \rightarrow \infty$ is reached with sufficient accuracy at finite screening radii $R$. The presence of the bound-state wave function in the matrix element strongly suppresses the contribution of the screened Coulomb interaction in high partial waves (i.e., two-baryon partial waves with orbital angular momentum $L \leqslant 6$ are sufficient for convergence). The other quantum-number-related cutoffs in the partial-wave dependence of the matrix element are the same as in Refs. [20,21], that is, $I \leqslant 4, \mathcal{J} \leqslant \frac{15}{2}$ for photoreactions, and $I \leqslant 3, \mathcal{J} \leqslant \frac{35}{2}$ for two-body electrodisintegration of ${ }^{3} \mathrm{He}$. All calculations include both total isospin $\mathcal{T}=\frac{1}{2}$ and $\mathcal{T}=\frac{3}{2}$ states. 


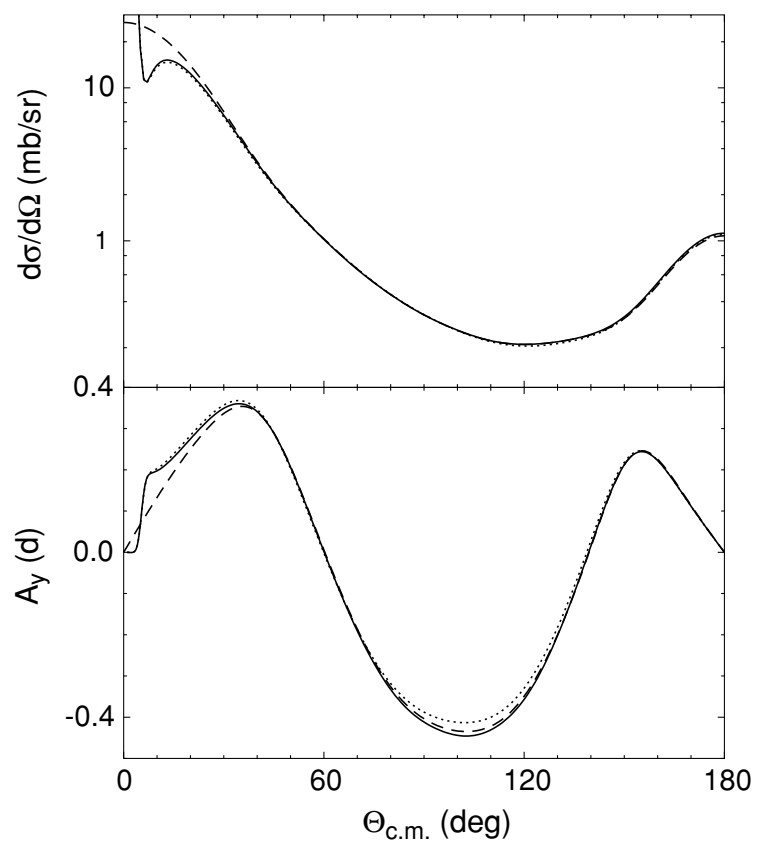

FIG. 7. Differential cross section and deuteron analyzing power $A_{y}(d)$ for $p d$ elastic scattering at $135-\mathrm{MeV}$ proton lab energy as functions of the c.m. scattering angle. Converged results of the present Coulomb treatment with $R=10 \mathrm{fm}$ given by solid curves are compared to the results calculated with no-Coulomb (dashed curves) and Coulomb externally corrected (dotted curves) approximations.

Figure 8 shows the convergence of our method with increasing screening radius $R$ for $p d$ radiative capture at $3-\mathrm{MeV}$ proton lab energy. We show the differential cross section and the deuteron tensor analyzing power $T_{21}$, which are the most critical observables in terms of convergence behavior. As in the case of $p p$ and $p d$ elastic scattering the convergence is impressive and becomes more rapid with increasing energy; it is quite comparable to $p d$ elastic scattering. The convergence with increasing screening radius $R$ is the same for two-body electrodisintegration of ${ }^{3} \mathrm{He}$; we therefore omit a corresponding figure.

\section{Conclusions on the practical implementation of the Coulomb interaction}

Using the described method we are able to include the Coulomb interaction between two protons in the description of hadronic and e.m. three-nucleon reactions in the $p d$ c.m. energy regime from about $1 \mathrm{MeV}$ up to the pion production threshold. The screening radius required for the convergence decreases with increasing energy. Whereas $R=20 \mathrm{fm}$ is required for energies around deuteron breakup threshold, the screening radius can be lowered to $R=10 \mathrm{fm}$ above $10-\mathrm{MeV}$ c.m. energy. In contrast, the screening radius has to be increased considerably when calculating extreme low-energy quantities, such as the $p d$ doublet scattering length, which at present is outside the reach of our adopted technique. However, the high-energy limit is imposed by the form of the hadronic interaction, which is applicable only below the pion production

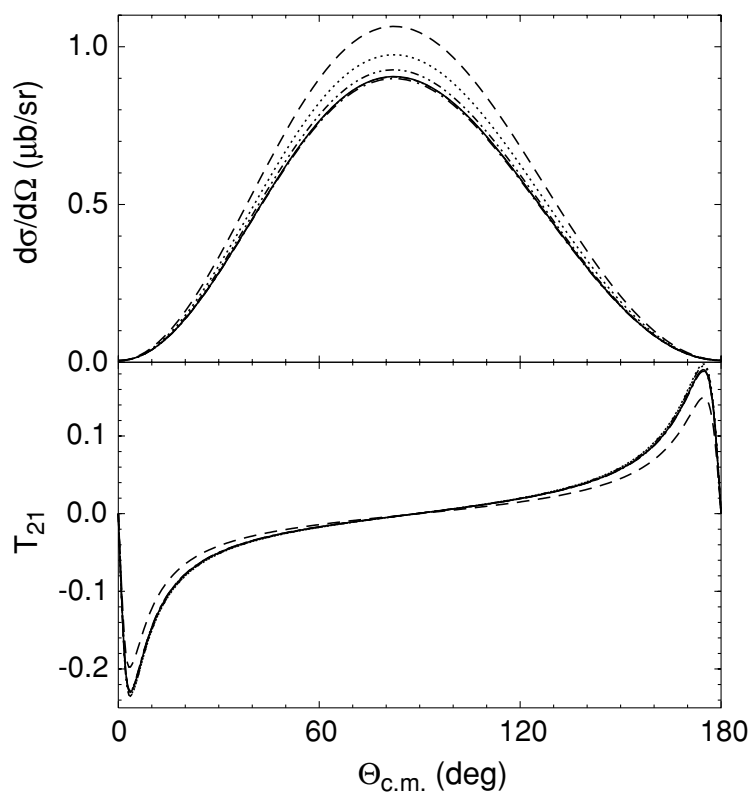

FIG. 8. Convergence of the differential cross section and of the deuteron analyzing power $T_{21}$ for $p d$ radiative capture at $3-\mathrm{MeV}$ proton lab energy with screening radius $R$. The observables are shown as functions of the c.m. scattering angle. The curves are explained in the caption of Fig. 5.

threshold. We notice no particular feature of the convergence when crossing the three-body breakup threshold. However, the paper does not treat the three-body breakup reactions yet.

As a side remark on the technique for solving the AGS equations, developed in Ref. [9], we find, in the context of the Coulomb interaction, the interpolation procedure for two-baryon transition matrices based on splines to be more convenient than the one based on the Chebyshev expansion.

\section{RESULTS}

We base our calculations on the two-baryon coupledchannel potential CD Bonn $+\Delta$ with and without the Coulomb interaction and use the CD Bonn potential with and without the Coulomb interaction as purely nucleonic reference. We use the charge and current operators of Refs. [20,21], which are appropriate for the underlying dynamics. We add relativistic corrections to the charge in the Siegert part of the operator when describing the photoreactions, an admittedly questionable procedure, but one entirely unrelated to the real issue of the Coulomb interaction in this paper.

Obviously, we have many more predictions than it is possible and wise to show. Therefore we make a judicious selection and present those predictions that illustrate the message we believe the results tell us. The readers, dissatisfied with our choice, are welcome to obtain the results for their favorite data from us.

Our predictions are dominantly based on the two-baryon coupled-channel potential CD Bonn $+\Delta$; its single virtual $\Delta$-isobar excitation yields, in the three-nucleon system, an effective three-nucleon force consistent with the two-nucleon 

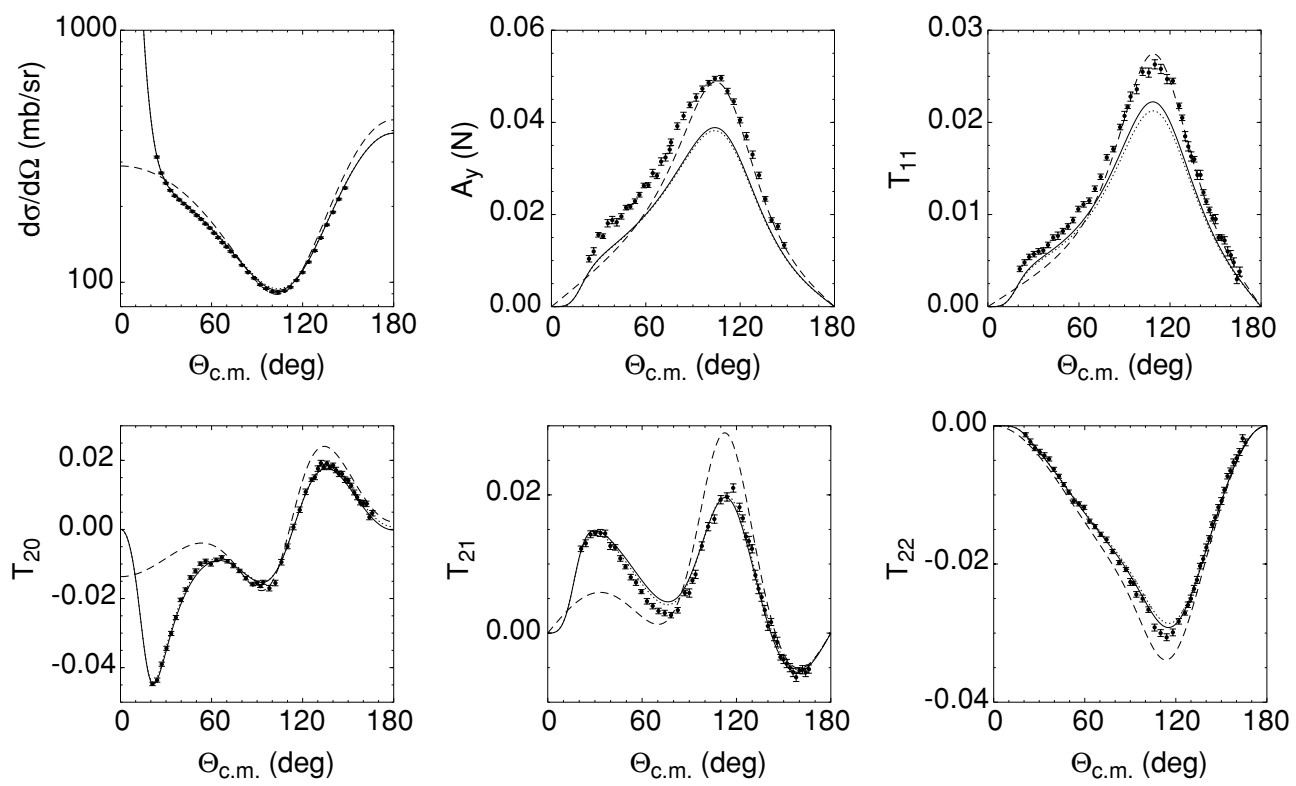

FIG. 9. Differential cross section and analyzing powers for $p d$ elastic scattering at 3-MeV proton lab energy as functions of the c.m. scattering angle. Results including $\Delta$-isobar excitation and the Coulomb interaction (solid curves) are compared to results without the Coulomb interaction (dashed curves). To better appreciate the size of the $\Delta$-isobar effect the purely nucleonic results including the Coulomb interaction are also shown (dotted curves). The experimental data are from Ref. [22].

interaction. $\Delta$-isobar effects increase the ${ }^{3} \mathrm{H}$ binding energy from 8.004 MeV for $\mathrm{CD}$ Bonn to $8.297 \mathrm{MeV}$ for $\mathrm{CD}$ Bonn $+\Delta$, the experimental value is $8.482 \mathrm{MeV}$. That binding energy increase has simultaneous beneficial effects on other bound state properties (e.g., on the charge radius) but those effects also appear in the $p d$ elastic scattering amplitude, especially in the three-nucleon $\mathcal{J}^{\Pi}=\frac{1}{2}^{+}$partial wave. The correlation between trinucleon binding and other low-energy observables is known as scaling. However, the Coulomb interaction also makes a significant contribution to trinucleon binding; the ${ }^{3} \mathrm{H}-{ }^{3} \mathrm{He}$ binding energy difference is $0.746 \mathrm{MeV}$ for CD Bonn and $0.756 \mathrm{MeV}$ for CD Bonn + $\Delta$, compared with the experimentally required value of 0.764 $\mathrm{MeV}$. This binding energy difference is dominantly due to the Coulomb repulsion between the protons in ${ }^{3} \mathrm{He}$; the contribution arising from the hadronic charge asymmetry is much smaller.

In three-nucleon scattering the $\Delta$ isobar therefore contributes to the scaling phenomenon at low energies, but it manifests itself more directly at higher energies when channel coupling becomes more probable. This section tries to explore the interplay between $\Delta$-isobar and Coulomb effects in the considered three-nucleon reactions.

\section{A. Elastic proton-deuteron scattering}

Figures 9 and 10 give characteristic low-energy results. As examples we show observables at $3-\mathrm{MeV}$ and $9-\mathrm{MeV}$ proton lab energy, respectively below and above deuteron breakup threshold. The Coulomb effect is quite appreciable at all scattering angles, but its relative importance decreases with increasing energy. In contrast, on the scale of the observed Coulomb effect, the $\Delta$-isobar effect is minute at those low energies. The inclusion of the Coulomb interaction is essential for a successful account of data for the spin-averaged differential cross section and for the deuteron tensor analyzing powers. However, its inclusion increases the discrepancy between theoretical predictions and experimental data in the peak region of proton and deuteron vector analyzing powers, the so-called $A_{y}$ puzzle. Our findings are consistent with the results of Refs. [1,3].

Figure 11 shows selected results at $135-\mathrm{MeV}$ proton lab energy. The Coulomb effect is confined to the forward direction, that is, to c.m. scattering angles smaller than $30^{\circ}$ where the $\Delta$-isobar effect is not visible. The $\Delta$-isobar effect shows up rather strongly in the region of the diffraction minimum, where its effect is beneficial and the Coulomb effect is gone. $\Delta$-isobar and Coulomb effects are nicely separated. Thus, the $\Delta$-isobar effect found previously [14] on the Sagara discrepancy and on spin observables remains essentially unchanged by the inclusion of the Coulomb interaction. The predictions of Fig. 11 are characteristic for all observables at higher energies.

\section{B. Proton-deuteron radiative capture}

The e.m. current operator is the standard choice of Ref. [20] supplemented by the relativistic one-nucleon charge corrections, also given in Ref. [20], which we found to be important for some spin observables even at low energies.

References [27,28] carried out corresponding realistic calculations for $p d$ radiative capture with different two-nucleon potentials and an irreducible three-nucleon force, but without relativistic one-nucleon charge corrections. The calculations 

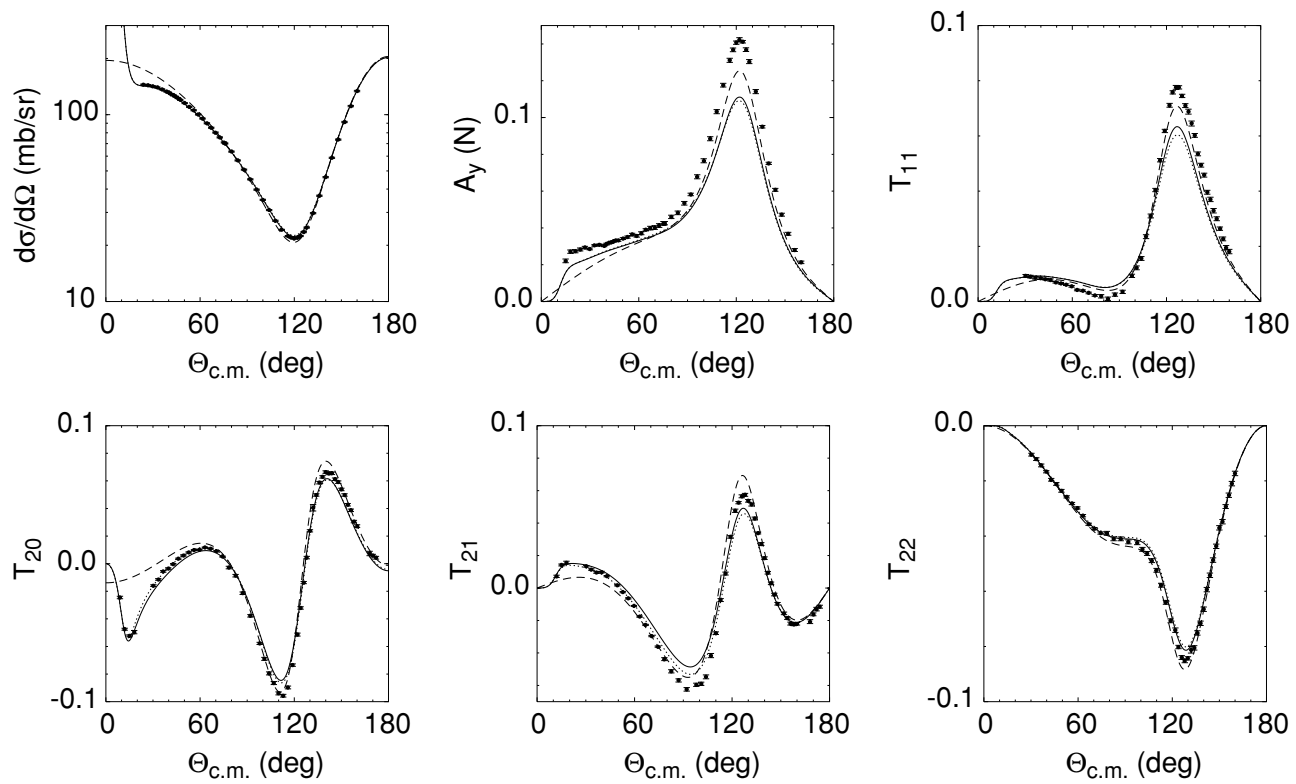

FIG. 10. Differential cross section and analyzing powers for $p d$ elastic scattering at 9-MeV proton lab energy as functions of the c.m. scattering angle. The curves are explained in the caption of Fig. 9. The experimental data are from Ref. [23].

of Ref. [27] take the Coulomb interaction fully into account but are limited to reactions below $10-\mathrm{MeV}$ c.m. energy. Reference [28] neglects the Coulomb interaction in the continuum states. When comparable, the results of this paper

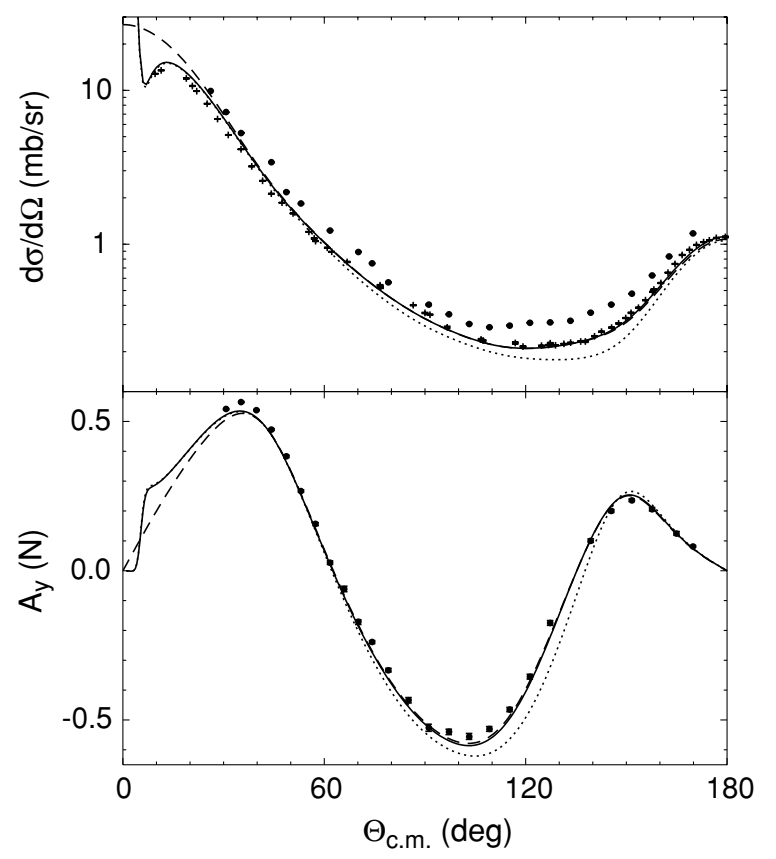

FIG. 11. Differential cross section and proton analyzing power $A_{y}(N)$ for $p d$ elastic scattering at $135 \mathrm{MeV}$ proton lab energy as functions of the c.m. scattering angle. The curves are explained in the caption of Fig. 9. The experimental data are from Ref. [24] (crosses) and from Ref. [25] (full circles) for the differential cross section and from Ref. [26] for the analyzing power. and those of Refs. [27,28] agree qualitatively; benchmark comparisons have not been done.

Figure 12 shows the Coulomb effect for $p d$ radiative capture at $3-\mathrm{MeV}$ proton lab energy. The Coulomb effect is most important for the differential cross section, which is reduced by about $20 \%$ and agrees rather well with the experimental data. In contrast, the spin observables show only a small Coulomb effect. The effect of relativistic one-nucleon charge corrections is entirely negligible for the differential cross section, but it is rather sizable and necessary for a satisfactory description of the data for the vector analyzing powers. Our results without relativistic one-nucleon charge corrections are consistent with the corresponding calculations of Ref. [27], which also fail to account for the vector analyzing power data. A moderate $\Delta$-isobar effect caused by scaling is visible around the peak of the differential cross section.

Selected deuteron analyzing powers at $17.5-\mathrm{MeV}$ deuteron lab energy with moderate Coulomb effects are shown in Fig. 13 together with the experimental data. Since the deuteron analyzing power $A_{y y}$ is rather flat between $40^{\circ}$ and $140^{\circ}$ according to Fig. 13, Fig. 14 focuses on the energy dependence of $A_{y y}$ at $90^{\circ}$ photon lab scattering angle. Clearly, our calculation accounts rather well for the known data of $A_{y y}$ in the entire deuteron lab energy region up to $95 \mathrm{MeV}$. However, a similar study for $T_{20}$ at $90^{\circ}$ (not shown here) indicates that the strong energy dependence of the low-energy data from TUNL [29] is not compatible with the present calculation. The rather good agreement with experimental data in Figs. 13 and 14 is obtained in general as an interplay of three considered effects, that is, the effects due to (i) the $\Delta$ isobar, (ii) the relativistic one-nucleon charge corrections, and (iii) the Coulomb interaction.

Figure 15 shows the differential cross section and the nucleon analyzing power for $p d$ radiative capture at 

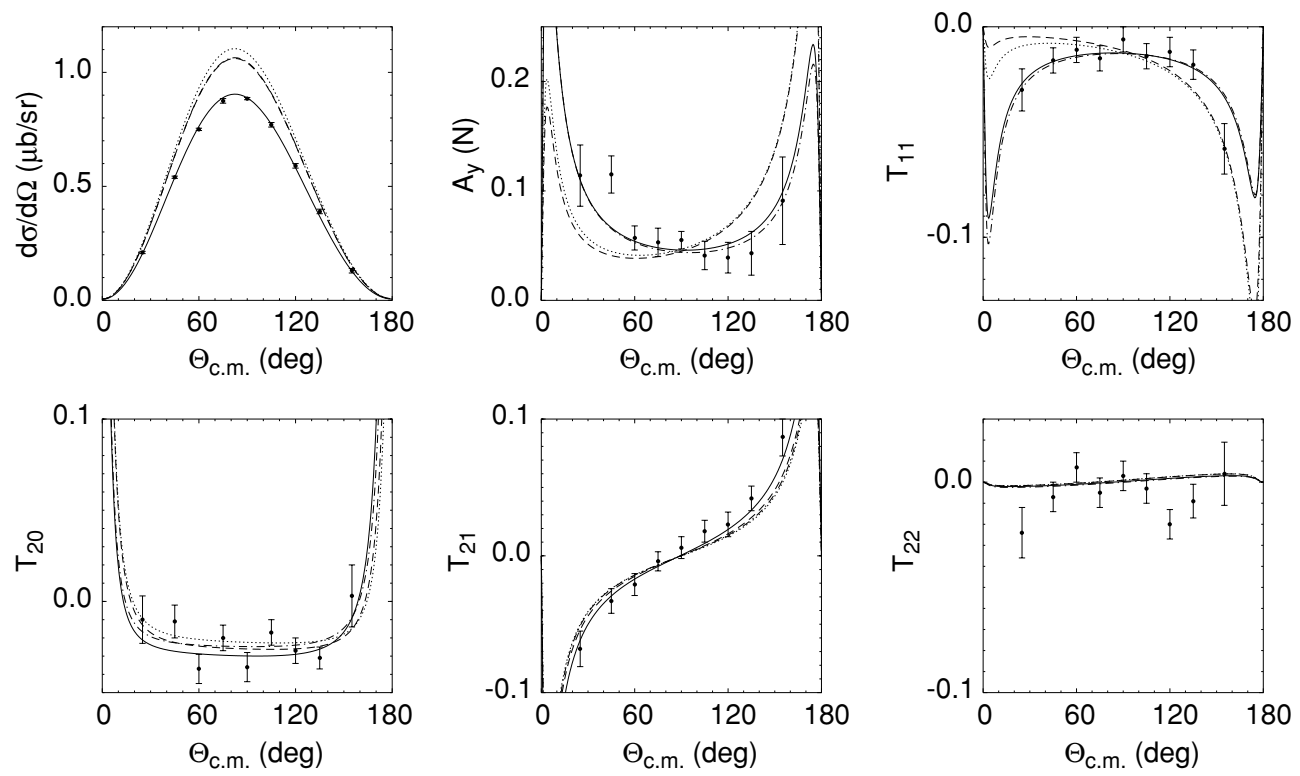

FIG. 12. Differential cross section and analyzing powers for $p d$ radiative capture at 3-MeV proton lab energy as functions of the photon c.m. scattering angle with respect to the direction of the proton. The results shown include, respectively, the Coulomb interaction, the relativistic one-nucleon charge corrections, and the $\Delta$-isobar excitation (solid curves); the relativistic one-nucleon charge corrections and the $\Delta$-isobar excitation (dashed-dotted curves); the $\Delta$-isobar excitation (dashed curves); and purely nucleonic results (dotted curves). The experimental data are from Ref. [30].

$150-\mathrm{MeV}$ nucleon lab energy where we previously found rather significant $\Delta$-isobar effects [20]. Even at this relatively high energy there is a visible, though small, Coulomb effect around the peak of the differential cross section. In addition, both observables show a sizable effect of the relativistic one-nucleon charge corrections.

Figures 12-15 also recall the non-Coulomb effects on observables to illustrate their interplay with the Coulomb interaction. In the differential cross section at $3-$ and $150-\mathrm{MeV}$ proton lab energy the $\Delta$ isobar plays different roles; at low energy the $\Delta$ isobar manifests itself through scaling caused by changed bound state properties, but at higher energies the explicit excitation of $\Delta$ channels in scattering becomes more predominant. We also note that relativistic one-nucleon charge corrections are important for proton and deuteron vector analyzing powers even at low energies; with increasing energy they become quite significant in general. The relativistic one-nucleon charge corrections are mostly
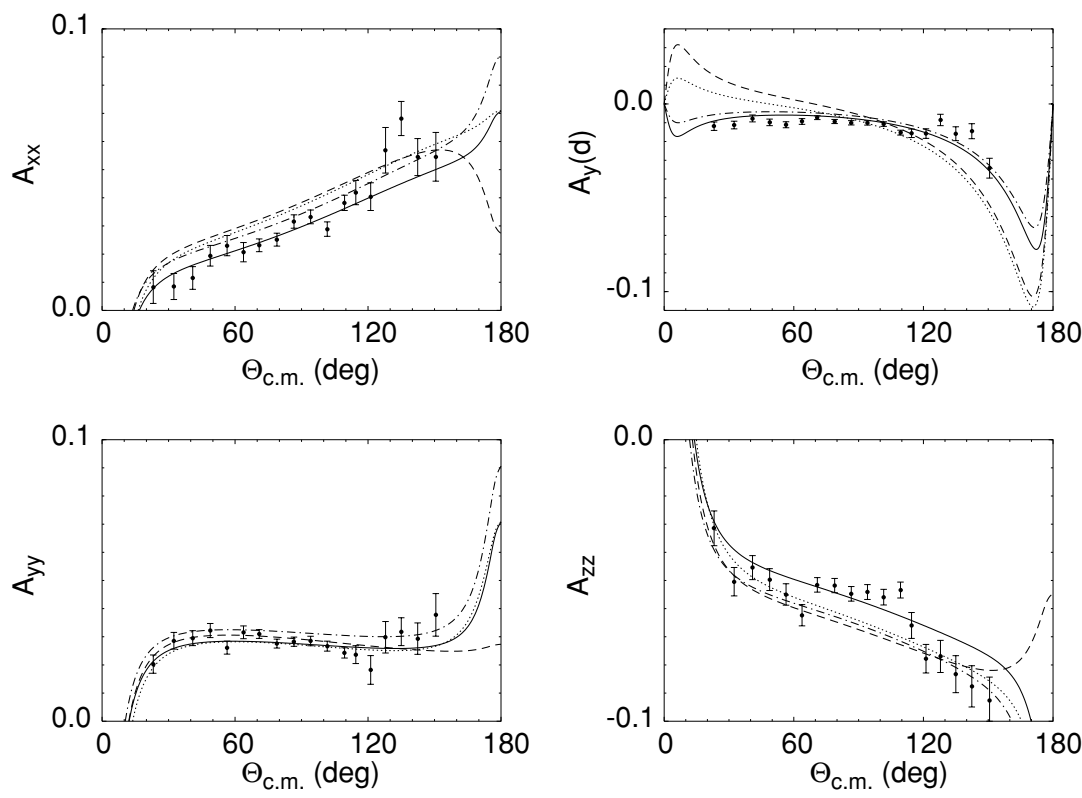

FIG. 13. Deuteron analyzing powers for $p d$ radiative capture at $17.5-\mathrm{MeV}$ deuteron lab energy as functions of the photon c.m. scattering angle with respect to the direction of the proton. The curves are explained in the caption of Fig. 12. The experimental data are from Ref. [31]. 


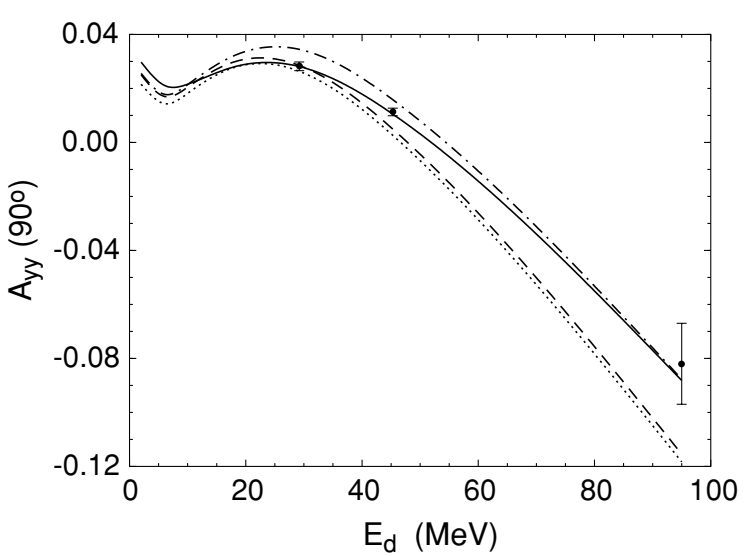

FIG. 14. Deuteron analyzing power $A_{y y}$ for $p d$ radiative capture at $90^{\circ}$ photon lab scattering angle as function of the deuteron lab energy. The curves are explained in the caption of Fig. 12. The experimental data are from Refs. [32,33].

beneficial for accounting for the experimental data, though their inclusion is not fully consistent with the underlying nonrelativistic hadronic dynamics.

\section{Two-body electrodisintegration of ${ }^{3} \mathrm{He}$}

The e.m. current operator is taken from Ref. [21]; compared to photo reactions, the relativistic one-nucleon charge corrections are less important and are therefore omitted. The Coulomb effect in the two-body electrodisintegration of ${ }^{3} \mathrm{He}$ depends on both energy and momentum transfer. We

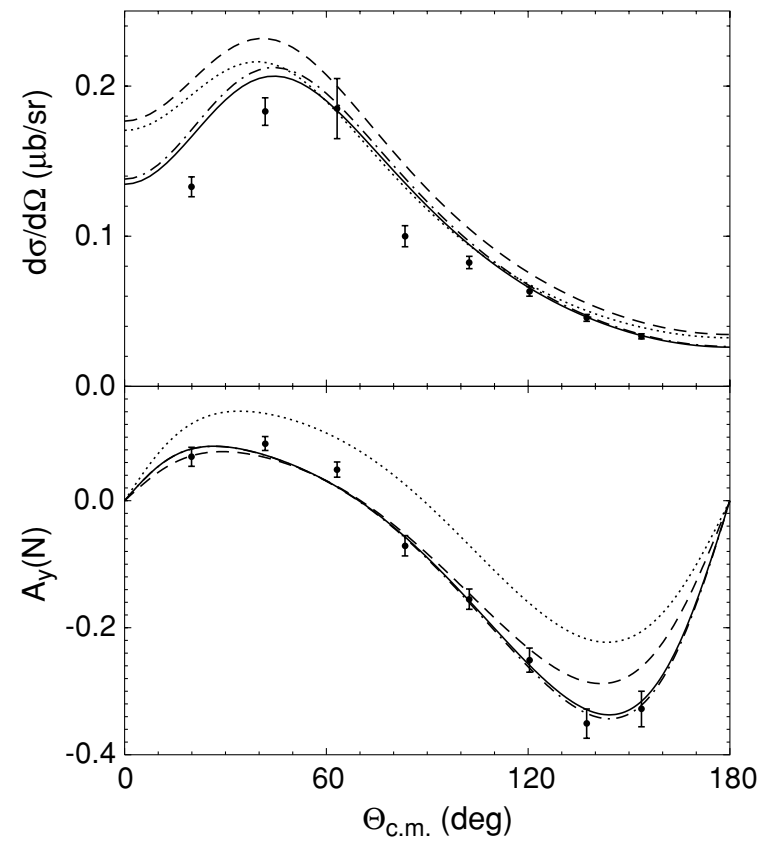

FIG. 15. Differential cross section and proton analyzing power $A_{y}(N)$ for $p d$ radiative capture at $150-\mathrm{MeV}$ proton lab energy as functions of the photon c.m. scattering angle with respect to the direction of the proton. The curves are explained in the caption of Fig. 12. The experimental data are from Ref. [34].
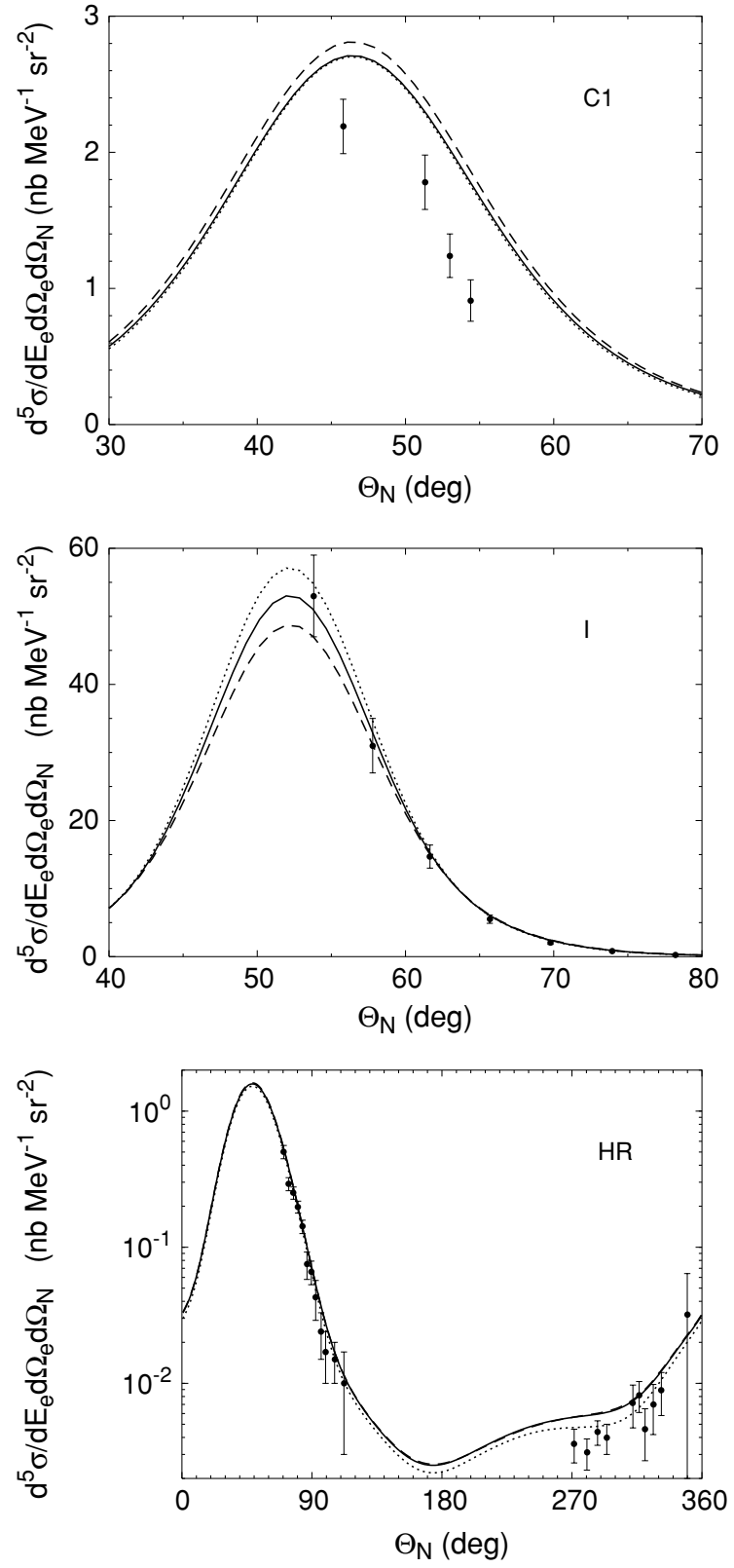

FIG. 16. Lab differential cross section for two-body electrodisintegration of ${ }^{3} \mathrm{He}$ as a function of the proton lab scattering angle. The electron lab energy, scattering angle, momentum, and energy transfer are $390 \mathrm{MeV}, 74.4^{\circ}, 434.8 \mathrm{MeV}$, and $66.1 \mathrm{MeV}$ for the reaction kinematics $\mathrm{C} 1 ; 527.9 \mathrm{MeV}, 52.2^{\circ}, 430.0 \mathrm{MeV}$, and $99.8 \mathrm{MeV}$ for the reaction kinematics I; and $390 \mathrm{MeV}, 39.7^{\circ}, 250.2 \mathrm{MeV}$, and 113.0 MeV for the reaction kinematics HR of Ref. [35], respectively. Results including $\Delta$-isobar excitation and the Coulomb interaction (solid curves) are compared to the results without the Coulomb interaction (dashed curves). To better appreciate the size of the $\Delta$-isobar effect the purely nucleonic results with the Coulomb interaction are also shown (dotted curves). The experimental data are from Ref. [35].

do not study that dependence in detail. We only show in Fig. 16 a sample result for the three reaction kinematics C1, I, and HR of Ref. [35]. The Coulomb effect on the $\mathrm{C} 1$ and I differential cross sections is visible in the peak, 
though in $\mathrm{C} 1$ it is small compared with the discrepancy between theoretical predictions and experimental data. In the HR differential cross section a Coulomb effect is not visible in the logarithmic scale of the plot, but instead a $\Delta$-isobar effect is observed at backward angles. Qualitatively our results without the Coulomb interaction agree well with the ones of Ref. [36].

\section{SUMMARY}

This paper shows how the Coulomb interaction between the charged baryons can be included in the momentumspace description of elastic proton-deuteron scattering and of related e.m. reactions using the screening and renormalization approach. The theoretical framework is the AGS integral equation [15]. The calculations are done on the same level of accuracy and sophistication as for the corresponding neutrondeuteron reactions. The conclusions of the paper refer to the developed technique and to the physics results obtained with that technique.

Technically, the idea of screening and renormalization is the one used in Refs. [11,12] and we rely on these works for mathematical rigor. However, our practical realization differs quite significantly from the one of Refs. [1,2] in the following ways:

(1) We use modern hadronic interactions, CD Bonn and CD Bonn $+\Delta$, in contrast to the low-rank separable potentials of Refs. [1,2]. Our use of the full potential requires the standard form of the three-particle equations, which differs from the quasiparticle approach of Refs. [1,2].

(2) We do not approximate the screened Coulomb transition matrix by the screened Coulomb potential.

(3) The quasiparticle approach of Refs. [1,2] treats the screened Coulomb potential between the protons without partial-wave expansion and therefore has no problems with the slow convergence of that expansion. Our solution of three-nucleon equations proceeds in partial-wave basis and therefore is faced with the slow partial-wave convergence of the Coulomb interaction between the charged baryons. However, we are able to obtain fully converged results by choosing a special form of the screening function and by using the perturbation theory of Ref. [17] for treating the screened Coulomb transition matrix in high partial waves. This would not be possible if we had used Yukawa screening as in Refs. [1,2] for two reasons: (a) The convergence with respect to screening would require much larger radii $R$; (b) the larger values of $R$ would necessitate the solution of the AGS equation with much higher angular momentum states.

(4) Our method for including the Coulomb interaction is efficient. Though the number of the isospin triplet partial waves to be taken into account is considerably higher than in the case without the Coulomb interaction, the required computing time increases only by a factor of 2 to 3 , owing to the use of the perturbation theory for high partial waves.

The obtained results are fully converged with respect to the screening and with respect to the quantum number cutoffs; they are therefore well checked for their validity. The employed technique gets cumbersome when approaching very low energies (i.e., $p d$ c.m. energies below $1 \mathrm{MeV}$ ) because of the need for very large screening radii. Thus, the calculation of the doublet scattering length for elastic $p d$ scattering at present, lies outside of our numerical reach, a barrier that does not exist for the coordinate-space techniques adopted in Refs. [3,4]. Nonetheless, we do not see any particular numerical problem when crossing the breakup threshold and going to higher energies where coordinate-space techniques are very hard to apply.

The present technique is not yet used for breakup itself, but such an extension is on its way.

Physicswise, the Coulomb effect in elastic $p d$ scattering is important at low energies for all kinematic regimes, but it gets confined to the forward direction at higher energies, whereas the effect mediated by the $\Delta$ isobar remains almost unmodified by the inclusion of the Coulomb interaction. In radiative $p d$ capture the Coulomb effect is important for low-energy differential cross sections and for some spin observables up to about 30-MeV proton lab energy; at higher energies there is still a visible Coulomb effect for some observables (e.g., in the peak of the differential cross section). In two-body electrodisintegration of ${ }^{3} \mathrm{He}$ the Coulomb effect appears not to be simply related to the internal excitation of the three-nucleon system. A thorough study of the dependence of the Coulomb effect on the energy and three-momentum transfer to the ${ }^{3} \mathrm{He}$ target is beyond the scope of this paper.

\section{ACKNOWLEDGMENTS}

The authors thank A. Kievsky and R. Lazauskas for providing benchmark results, K. Sagara for providing the experimental data, and S. Oryu and A. Stadler for useful discussions. A.D. is supported by the FCT Grant SFRH/BPD/14801/2003 and by the DFG Grant Sa 247/25, P.U.S. in part by the DFG Grant Sa 247/25, and A.C.F. in part by the FCT Grant POCTI/FNU/37280/2001.
[1] E. O. Alt, A. M. Mukhamedzhanov, M. M. Nishonov, and A. I. Sattarov, Phys. Rev. C 65, 064613 (2002).

[2] G. H. Berthold, A. Stadler, and H. Zankel, Phys. Rev. C 41, 1365 (1990).

[3] A. Kievsky, M. Viviani, and S. Rosati, Phys. Rev. C 64, 024002 (2001).

[4] C. R. Chen, J. L. Friar, and G. L. Payne, Few-Body Syst. 31, 13 (2001).
[5] V. M. Suslov and B. Vlahovic, Phys. Rev. C 69, 044003 (2004).

[6] E. O. Alt, S. B. Levin, and S. L. Yakovlev, Phys. Rev. C 69, 034002 (2004).

[7] S. Oryu, Few-Body Syst. 34, 113 (2004); S. Oryu and S. Gojuki, Prog. Theor. Phys. Suppl. 154, 285 (2004)

[8] W. Glöckle, H. Witała, D. Hüber, H. Kamada, and J. Golak, Phys. Rep. 274, 107 (1996). 
[9] A. Deltuva, K. Chmielewski, and P. U. Sauer, Phys. Rev. C 67, 034001 (2003).

[10] P. Doleschall, W. Grüebler, V. Konig, P. A. Schmelzbach, F. Sperisen, and B. Jenny, Nucl. Phys. A380, 72 (1982).

[11] J. R. Taylor, Nuovo Cimento B 23, 313 (1974); M. D. Semon and J. R. Taylor, ibid. A26, 48 (1975).

[12] E. O. Alt, W. Sandhas, and H. Ziegelmann, Phys. Rev. C 17, 1981 (1978); E. O. Alt and W. Sandhas, ibid. 21, 1733 (1980).

[13] R. Machleidt, Phys. Rev. C 63, 024001 (2001).

[14] A. Deltuva, R. Machleidt, and P. U. Sauer, Phys. Rev. C 68, 024005 (2003).

[15] E. O. Alt, P. Grassberger, and W. Sandhas, Nucl. Phys. B2, 167 (1967).

[16] L. S. Rodberg and R. M. Thaler, Introduction to the Quantum Theory of Scattering (Academic Press, New York, 1967).

[17] A. Deltuva, K. Chmielewski, and P. U. Sauer, Phys. Rev. C 67, 054004 (2003).

[18] E. O. Alt, A. M. Mukhamedzhanov, and A. I. Sattarov, Phys. Rev. Lett. 81, 4820 (1998).

[19] A. Deltuva, A. C. Fonseca, A. Kievsky, S. Rosati, P. U. Sauer, and M. Viviani, Phys. Rev. C (to be published), nucl-th/0503015.

[20] A. Deltuva, L. P. Yuan, J. Adam, Jr., A. C. Fonseca, and P. U. Sauer, Phys. Rev. C 69, 034004 (2004).

[21] A. Deltuva, L. P. Yuan, J. Adam, Jr., and P. U. Sauer, Phys. Rev. C 70, 034004 (2004).

[22] S. Shimizu, K. Sagara, H. Nakamura, K. Maeda, T. Miwa, N. Nishimori, S. Ueno, T. Nakashima, and S. Morinobu, Phys. Rev. C 52, 1193 (1995).

[23] K. Sagara, H. Oguri, S. Shimizu, K. Maeda, H. Nakamura, T. Nakashima, and S. Morinobu, Phys. Rev. C 50, 576 (1994); K. Sagara (private communication).
[24] K. Sekiguchi H. Sakai, H. Witała, W. Glöckle, J. Golak, M. Hatano, H. Kamada, H. Kato, Y. Maeda, and J. Nishikawa et al., Phys. Rev. C 65, 034003 (2002).

[25] K. Ermisch, H. R. Amir-Ahmadi, A. M. van den Berg, R. Castelijns, B. Davids, E. Epelbaum, E. van Garderen, W. Glöckle, and J. Golak et al., Phys. Rev. C 68, 051001(R) (2003).

[26] K. Ermisch, A. M. van den Berg, R. Bieber, W. Glöckle, J. Golak, M. Hagemann, V. M. Hannen, M. N. Harakeh, M. A. de Huu, and N. Kalantar-Nayestanaki et al., Phys. Rev. Lett. 86, 5862 (2001).

[27] L. E. Marcucci, M. Viviani, R. Schiavilla, A. Kievsky, and S. Rosati, nucl-th/0411083.

[28] J. Golak, H. Kamada, H. Witała, W. Glöckle, J. Kuros, R. Skibiński, V. V. Kotlyar, K. Sagara, and H. Akiyoshi, Phys. Rev. C 62, 054005 (2000).

[29] G. J. Schmid, R. M. Chasteler, H. R. Weller, D. R. Tilley, A. C. Fonseca, and D. R. Lehman, Phys. Rev. C 53, 35 (1996).

[30] M. K. Smith and L. D. Knutson, Phys. Rev. Lett. 82, 4591 (1999).

[31] H. Akiyoshi, K. Sagara, S. Ueno, N. Nishimori, T. Fujita, K. Maeda, H. Nakamura, and T. Nakashima, Phys. Rev. C 64, 034001 (2001).

[32] J. Jourdan et al., Phys. Lett. B162, 269 (1985).

[33] W. K. Pitts et al., Phys. Rev. C 37, 1 (1988).

[34] M. A. Pickar, H. J. Karwowski, J. D. Brown, J. R. Hall, M. Hugi, R. E. Pollock, V. R. Cupps, M. Fatyga, and A. D. Bacher, Phys. Rev. C 35, 37 (1987).

[35] E. Jans et al., Nucl. Phys. A475, 687 (1987); E. Jans (private communication).

[36] S. Ishikawa, H. Kamada, W. Glöckle, J. Golak, and H. Witała, Nuovo Cimento A 107, 305 (1994). 